\title{
The Galactic Environment of the Sun: Interstellar Material Inside and Outside of the Heliosphere
}

\author{
P.C. Frisch • M. Bzowski • E. Grün • V. Izmodenov • H. Krüger · J.L. Linsky • \\ D.J. McComas · E. Möbius · S. Redfield • N. Schwadron · R. Shelton · J.D. Slavin • \\ B.E. Wood
}

Received: 30 July 2008 / Accepted: 24 March 2009 / Published online: 14 May 2009

(C) Springer Science+Business Media B.V. 2009

\begin{abstract}
Interstellar material (ISMa) is observed both inside and outside of the heliosphere. Relating these diverse sets of ISMa data provides a richer understanding of both the interstellar medium and the heliosphere. The galactic environment of the Sun is dominated by warm, low-density, partially ionized interstellar material consisting of atoms and dust grains. The properties of the heliosphere are dependent on the pressure, composition, radiation field, ionization, and magnetic field of ambient ISMa. The very low-density interior of the Local Bubble, combined with an expanding superbubble shell associated with star formation in the Scorpius-Centaurus Association, dominate the properties of the local interstellar medium (LISM). Once the heliosphere boundaries and interaction mechanisms are understood, interstellar gas, dust, pickup ions, and anomalous cosmic rays inside of the heliosphere can be directly compared to ISMa outside of the heliosphere. Our understanding of ISMa at the Sun is further enriched when the circumheliospheric interstellar material is compared to observations of other nearby ISMa and the overall context of our galactic environment. The IBEX mission will map the interaction region between the heliosphere and ISMa, and improve the accuracy of comparisons between ISMa inside and outside the heliosphere.
\end{abstract}

\footnotetext{
P.C. Frisch $(\varangle)$

University of Chicago, Chicago, IL, USA

e-mail: frisch@oddjob.uchicago.edu

M. Bzowski

Space Research Centre PAS, Warsaw, Poland

e-mail: bzowski@cbk.waw.pl

E. Grün

Max-Planck-Institut fuer Kernphysik, Heidelberg, Germany

e-mail: Eberhard.Gruen@mpi-hd.mpg.de
}

V. Izmodenov

Moscow State University and Space Research Institute RAS, Moscow, Russia e-mail: izmod@ipmnet.ru

H. Krüger

Max-Planck-Institut fuer Sonnensystemforschung, Katlenburg-Lindau, Germany

e-mail: krueger@mps.mpg.de 
Keywords Interstellar material $\cdot$ Heliosphere $\cdot$ Local bubble

\title{
1 Introduction
}

The heliosphere is flooded with low-density interstellar neutral gas and dust that have a flow velocity of $\sim 95,000 \mathrm{~km} \mathrm{~h}^{-1}$ and an upstream direction towards the Scorpius-Centaurus Association. Both raw interstellar particles and byproducts of the interaction of interstellar neutrals with the solar wind plasma are found within 1 AU of the Sun. These particles provide an in situ sample of the cosmos. Voyager 1 (V1) crossed the solar wind termination shock at ecliptic latitude $\beta=+35^{\circ}$ and distance 94 AU (Stone et al. 2005, e.g.), while Voyager 2 (V2) crossed the termination shock at $\beta=-31^{\circ}$ and distance 84 AU (e.g. Stone et al. 2008; Richardson et al. 2008; Gurnett and Kurth 2008; Burlaga et al. 2008, Fig. 1). The two Voyager spacecraft provide in situ data on both solar wind and interstellar particle populations in the inner heliosheath regions. The IBEX mission (McComas et al. 2004a, 2005, 2006, 2009, this issue), will add to these data by mapping the interaction between interstellar neutrals and the solar wind using observations of energetic neutral atoms (ENAs) with energies of $10 \mathrm{eV}-6 \mathrm{keV}$, formed from charge exchange between $\mathrm{H}^{\circ}$ and $\mathrm{H}^{+}$. The ENA data will constrain the filtration of interstellar neutrals entering the heliosphere. Building a self-consistent picture of the interaction between the heliosphere and interstellar material (ISMa) from these diverse data requires knowledge of the interstellar cloud surrounding the heliosphere. The surrounding cloud is part of a dynamical flow of interstellar gas and

\author{
J.L. Linsky \\ University of Colorado and NIST, Boulder, CO, USA \\ e-mail: jlinsky@jila.colorado.edu \\ D.J. McComas \\ Southwest Research Institute, San Antonio, TX, USA \\ e-mail: DMcComas@swri.edu \\ E. Möbius \\ University of New Hampshire, Durham, NH, USA \\ e-mail: eberhard.moebius@unh.edu
}

S. Redfield

Wesleyan University, Middletown, CT, USA

e-mail: sredfield@wesleyan.edu

N. Schwadron

Boston University, Boston, MA, USA

e-mail: nathanas@bu.edu

R. Shelton

University of Georgia, Athens, Georgia

e-mail: rls@physast.uga.edu

J.D. Slavin

SAO-Harvard, Cambridge, MA, USA

e-mail: jslavin@cfa.harvard.edu

B.E. Wood

Naval Research Lab, Washington, DC, USA

e-mail: brian.wood@nrl.navy.mil 


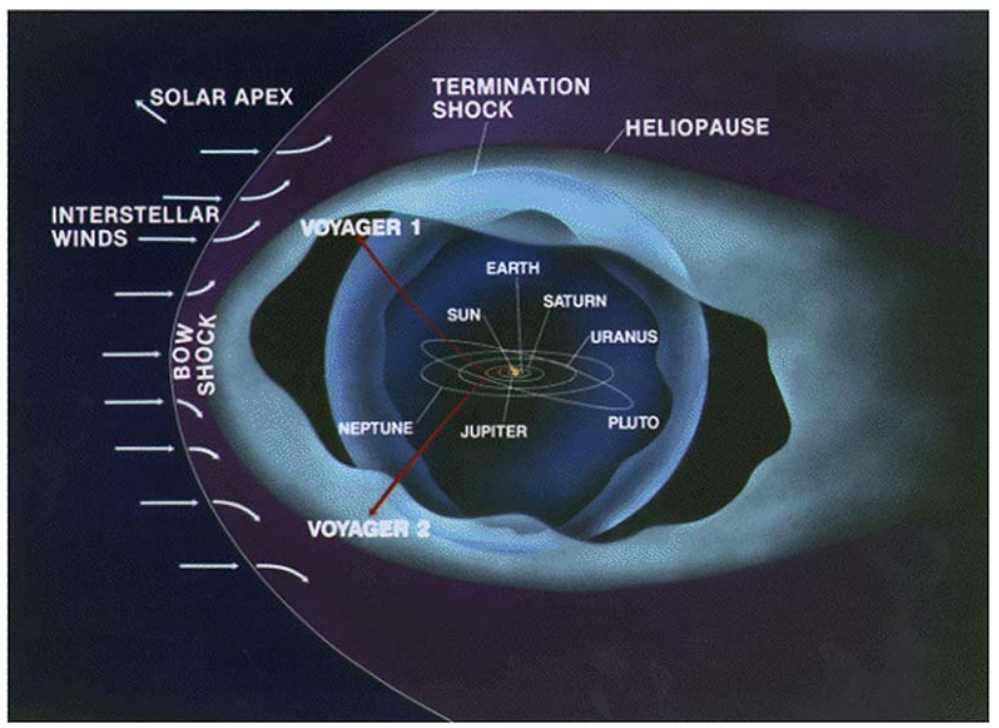

Fig. 1 Cartoon of the heliosphere showing the V1 and V2 trajectories. Interstellar gas flows into the heliosphere from the left. The solar wind termination shock, the heliopause, which is the contact discontinuity between solar wind and interstellar plasmas, and the bow shock are indicated. The ram pressures of the interstellar material, including the interstellar magnetic field, and the solar wind dominate the heliosphere configuration

dust through space. Once we know the physical properties of this flow, we will know the past and future galactic environment of the Sun. Extreme variations in the properties of the interstellar medium surrounding the Sun will have an extreme effect on the heliosphere, the flux of galactic cosmic rays (GCRs) onto the Earth, and possibly the terrestrial climate (Müller et al. 2006; Fahr et al. 2006; Florinski and Zank 2006; Frisch 2006). IBEX will provide key observations of the heliosphere response to our galactic environment.

The space age is old enough that we now have observations of ISMa inside of the heliosphere spanning almost four decades, and these data suggest that the cloud containing the circumheliospheric interstellar medium (CHISM) is homogeneous over spatial scales of at least $\sim 115 \mathrm{AU}$ in the downwind direction of the interstellar flow. The CHISM is defined as the parent cloud of the interstellar gas and dust flowing into the heliosphere. The CHISM appears to be at the edge of the Local Interstellar Cloud (LIC), which belongs to a flow of local interstellar medium (LISM, distance $\leq 40-70 \mathrm{pc}$ ) through space (Sect. 5.1). Interstellar gas was first discovered inside of the heliosphere through broad-band observations of the fluorescence of solar Ly $\alpha$ and $584 \AA$ emissions from interplanetary $\mathrm{H}^{\circ}$ and $\mathrm{He}^{\circ}$, respectively (Thomas and Krassa 1971; Bertaux and Blamont 1971; Weller and Meier 1974, 1981; Adams and Frisch 1977; Ajello et al. 1987), and the importance of these data was quickly recognized (e.g. Holzer 1972; Fahr 1974; Wallis 1975; Thomas 1978). Copernicus made the first spectrum of the Ly $\alpha$ backscattered radiation and showed that interstellar gas inside of the solar system has a velocity similar to the velocity of interstellar clouds in the solar vicinity (Adams and Frisch 1977). The interplanetary Ly $\alpha$ glow from interstellar $\mathrm{H}^{\circ}$ inside of the heliosphere provided the first glimpse that the CHISM is warm and tenuous, and quite different from the galactic cold dense $\mathrm{H}^{\circ}$ that dominates the $\mathrm{H}^{\circ} 21-\mathrm{cm}$ hyperfine emission sky. The difference between the observed inflow velocities of $\mathrm{H}^{\circ}$ and $\mathrm{He}^{\circ}$ was later shown to be due partly to secondary $\mathrm{H}^{\circ}$ production from charge exchange between interstellar $\mathrm{H}^{\circ}$ 
and interstellar protons near the heliosphere boundaries, as well as solar radiation pressure (Sect. 2.1.2). Slavin and Frisch (2008, SF08) have compared the spectra of the $\mathrm{H}^{\circ} \mathrm{Ly} \alpha$ interplanetary glow observed by Copernicus during 1975 (Adams and Frisch 1977) with spectra collected by the Hubble Space Telescope (HST) during the 1990s (Clarke et al. 1998), and concluded that the velocity of the interstellar cloud feeding $\mathrm{H}^{\circ}$ into the heliosphere has not changed substantially over the intervening two decades, and that the circumheliospheric interstellar cloud extends at least $\sim 115 \mathrm{AU}$ in the interstellar downwind direction. Ulysses observations of interstellar dust inside of the heliosphere during 1992-2002 can be fit with a constant dust density in the CHISM, also providing support for a homogeneous CHISM over scale sizes of $\sim 55$ AU (Landgraf 2000).

The Sun resides in a region of space with very low average interstellar densities, the Local Bubble (LB), formed by ISMa associated with the Gould's Belt ring of young stars. Gould's Belt appears to belong to the 'Orion spur', protruding $\sim 1 \mathrm{kpc}$ from the leading (convex) edge of the Sagittarius spiral arm into the adjacent interarm region (Frisch 2008a). The galactic setting of the Sun is dominated by the LB void in ISMa, and the LIC of which the CHISM is the nearest part. The LIC belongs to a low-density flow of interstellar material away from the Scorpius-Ophiuchus Association (Frisch 1981). Figure 2 shows the solar location inside of the LB void; the molecular clouds that border this void are associated with regions of star formation (for an alternate LB representation see Sfeir et al. 1999).

This chapter presents the data that help us determine the properties of the interstellar cloud that now surrounds the heliosphere. We start with the discussion of ISMa deep inside of the heliosphere (Sect. 2), including both primary and processed interstellar neutrals (Sect. 2.1). This includes $\mathrm{He}^{\mathrm{o}}$ (Sect. 2.1.1), $\mathrm{H}^{\circ}$ (Sect. 2.1.2), pickup ions (PUIs, Sect. 2.2) consisting of ionized interstellar neutrals, and anomalous cosmic rays (ACRs, Sect. 3.1) consisting of accelerated PUIs. Larger interstellar dust grains (ISDGs) also flow through the heliosphere, penetrating to the Earth and offering a new glimpse of stardust (Sect. 2.3). We then discuss the data that trace the boundary regions of the heliosphere (Sect. 3), including the increased velocity dispersion of interstellar $\mathrm{H}^{\circ}$ in the hydrogen wall (Sect. 3.2) and deficit of small interstellar dust grains inside the heliosphere (Sect. 3.3), both caused by charged ISMa pushed against the magnetized plasma in the outer heliosheath. The small dust grains in this region also appear to trace the direction of the interstellar magnetic field (ISMF) at the Sun, giving us clues about the origin of the LIC in a superbubble shell (Sect. 3.4). We then show that the properties of our immediate galactic environment and the heliosphere boundary conditions are affected by the Sun's location inside of the Local Bubble (Sect. 4), which controls the interstellar radiation field (ISRF) and ionizes nearby ISMa (Sect. 4.1). An important part of understanding the ISRF at the Sun is to first understand foreground contamination of the LB X-ray emission by charge-exchange between interstellar neutrals and the solar wind (Sect. 4.2). Once the interstellar radiation field is understood, the detailed boundary conditions of the heliosphere can be reconstructed using radiative transfer models of the LIC and observations of ISMa inside and outside of the heliosphere (Sect. 4.3). We then look at the family of nearby interstellar clouds, and find a best fit velocity that indicates a flow of ISMa from an upwind direction towards the Scorpius-Centaurus Association (Sect. 5). The overall properties of this dynamical flow of gas within $30 \mathrm{pc}$ have been known for some time, but more detailed information on the velocity structure and physical properties are now available (5.1); we discuss the origin (5.2) and physical properties (Sect. 5.3) of these clouds.

The acronyms used here are summarized at the end of the chapter. Note that the terms LIC and CHISM are not interchangable. The CHISM is the source of the interstellar gas and dust flowing into the heliosphere, whereas the LIC is the interstellar 'cloud' reconstructed 


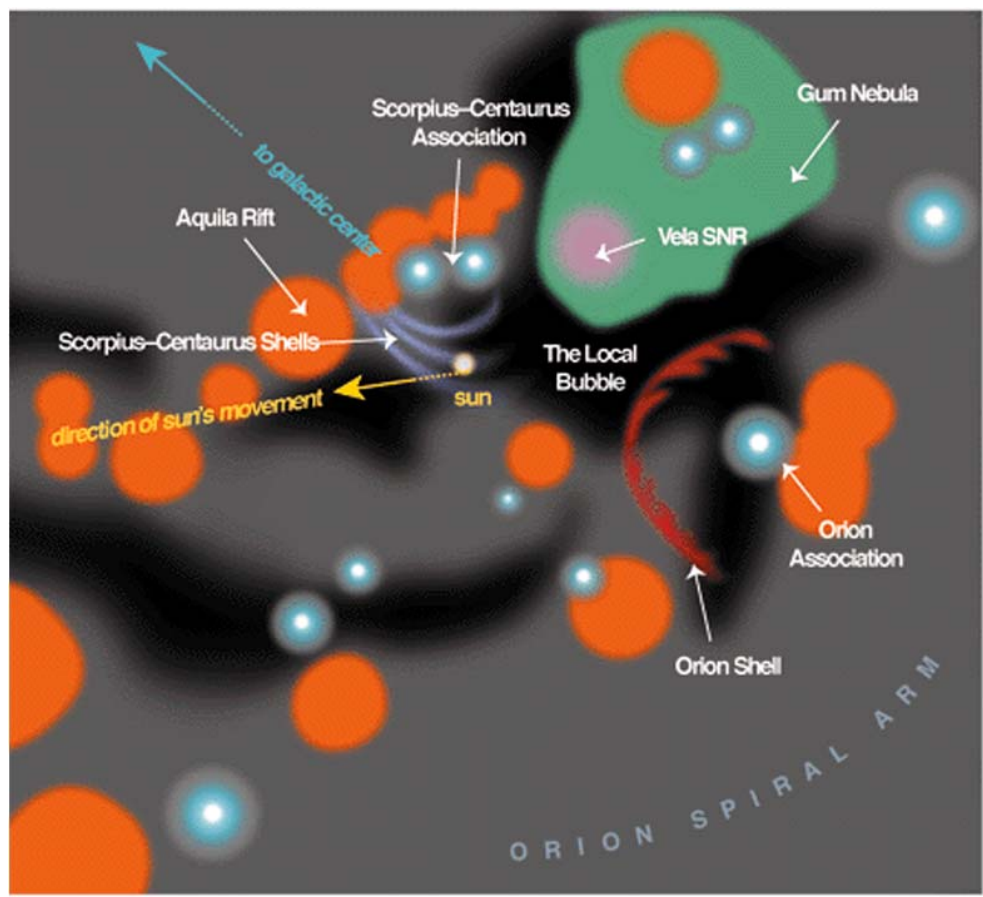

molecular douds

dffuse gas

Fig. 2 The galactic setting of the Sun is shown out to distances of 400-500 pc, as viewed from a high latitude sightline in the second galactic quadrant. The galactic center is to the upper left (blue arrow), and the anticenter is below Orion (lower-middle, right). The superbubble shells expanding away from the Scorpius-Centaurus Association (upper middle) are shown as blue arcs; the CHISM is part of the S1 subshell of the Loop I superbubble (Sect. 3.4). The solar apex motion, and our location inside of the rim of the S1 shell, is shown in yellow. (The solar apex motion is defined as the velocity of the Sun with respect to the local standard of rest (LSR), e.g. with respect to the mean kinetic motion of a set of nearby cool, old, stars moving in tandem around the center of the galaxy.) The large orange circles show the locations of molecular clouds (CO data) bordering the Local Bubble. The blue dots show the locations of nearby star-forming associations (based on Hipparcos data). The S1 and S2 magnetic shells (Wolleben 2007) merge where Loop I (gray arcs) encounters the very dusty Aquila Rift. The Orion spur is often denoted the "Orion spiral arm", as is done in this figure. "Spurs" form from instabilities on magnetized, self-gravitating spiral arms (Kim and Ostriker 2002). The overall relation between the Orion Spur and the Sagittarius spiral arm can be seen in the reddening data in Fig. 10 of Lucke (1978). The Sagittarius spiral arm is approximately $1 \mathrm{kpc}$ from the Sun in the direction of the galactic center (e.g. Russeil 2003). Figure copyrighted by American Scientist (Frisch 2000)

from the kinematics of an arbitrary set of velocity components towards nearby stars. The original definition of the LIC was intended to identify the interstellar cloud of which the CHISM is a part, but that definition is no longer universally accepted (Sects. 5.1, 5.1.2).

\section{Interstellar Matter inside of the Heliosphere}

Interstellar material inside of the heliosphere consists of neutral interstellar gas and large weakly charged dust grains. These atoms and grains interact with the solar wind, solar gravity, and the solar radiation field, and provide an in situ sample of the CHISM. These interstellar neutrals also form the parent population of PUIs and ACRs observed inside of the 
heliosphere. Additional interstellar populations observed at $1 \mathrm{AU}$, but not discussed in detail here, include interstellar micrometeorites and galactic cosmic rays (GCRs). The interstellar micrometeorites have masses $\sim 10^{-9.7} \mathrm{~kg}$ and velocities larger than the solar system escape velocity, indicating an origin outside of the heliosphere (see Baggaley 2000; Landgraf et al. 2000). Galactic cosmic rays are measured over 18 orders of magnitude in energy, and provide an in situ sample of stellar nucleosynthesis products as well as shock-accelerated interstellar material. Low energy GCRs are excluded from the heliosphere, while more energetic ions arrive at the surface of the Earth. Galastic cosmic rays are not discussed further, but excellent overviews of the origin and abundances of GCRs can be found elsewhere (e.g. Wiedenbeck et al. 2007).

\subsection{Interstellar Neutrals inside the Heliosphere}

Neutral interstellar atoms trace the CHISM neutrality at the point of entry to the heliosphere. These neutrals are observed through solar backscattered emission $\left(\mathrm{H}^{\circ}\right.$ and $\left.\mathrm{He}^{\circ}\right)$, as PUIs formed by interstellar neutrals ionized inside of the heliosphere and subsequently convected outwards by the solar wind, and as ACRs formed from accelerated PUIs (Sect. 3.1). Interstellar $\mathrm{He}^{\mathrm{o}}$ inside of the heliosphere provides the benchmark data on the velocity and temperature of the CHISM.

\subsubsection{Helium}

Neutral interstellar helium flows freely through the outer heliosphere, with $\leq 2 \%$ filtration through charge exchange with $\mathrm{H}^{+}$, and is ionized by photoionization and electron ionization inside of the Earth's orbit (e.g. see Cummings et al. 2002; Müller et al. 2004, for $\mathrm{He}^{\mathrm{o}}$ filtration factors). Our knowledge of interstellar $\mathrm{He}^{\mathrm{o}}$ inside the heliosphere is summarized from the present-day viewpoint by Möbius et al. (2004), and the classical viewpoint by Fahr (1974). Early observations of $\mathrm{H}^{\circ}$ and $\mathrm{He}^{\circ}$ inside of the heliosphere found ratios $\mathrm{H}^{\circ} / \mathrm{He}^{\mathrm{o}} \sim$ 6-7 (Ajello et al. 1987; Chassefiere et al. 1986) in contrast to the cosmic value $\mathrm{H} / \mathrm{He}=10$. The relatively high heliospheric abundance of $\mathrm{He}$ is now known to be due partly to the high $\mathrm{H}^{\circ}$ loss rate from charge exchange in the outer heliosphere, counteracted by the hard local interstellar radiation field that is more efficient at ionizing $\mathrm{He}^{\circ}$ than $\mathrm{H}^{\circ}$ in the low-density surrounding cloud (Sect. 4.3). Photoionization and electron impact ionization dominate $\mathrm{He}^{\mathrm{O}}$ loss mechanisms in the inner heliosphere. The trajectory of interstellar $\mathrm{He}^{\mathrm{o}}$ inside of the heliosphere is unaffected by radiation pressure and charge exchange. Neutral interstellar He collects in a gravitational focusing cone extending $\sim 8 \mathrm{AU}$ downwind of the Sun (Sect. 2.2), which the Earth traverses early December each year (Fig. 3). Cone properties are derived self-consistently from He pickup ions and $\mathrm{He}^{\mathrm{o}}$ backscattered $584 \AA$ emission, for some combination of photoionization and electron impact ionization rates (Möbius et al. 2004, Sect. 2.2).

Interstellar helium has been observed in the heliosphere in four different ways, through fluorescence of solar $584 \AA$ emissions (Weller and Meier 1974; Vallerga et al. 2004), direct particle counting by the GAS detector on Ulysses (Witte 2004), as PUIs formed from ionized interstellar He convected radially outwards by the solar wind (Sect. 2.2, Möbius et al. 1985; Geiss et al. 1995; Gloeckler et al. 2000), and as ACRs generated from accelerated PUIs (Sect. 2.2, e.g. Cummings et al. 2002). Interstellar helium is primarily neutral at the Earth, becoming ionized by photoionization and electron impact ionization within $\sim 0.5 \mathrm{AU}$ of the Sun. Möbius et al. (2004) have compared the He results from pickup ions, extreme ultraviolet (EUV) fluorescence, and direct particle detections, to obtain weighted mean values for 


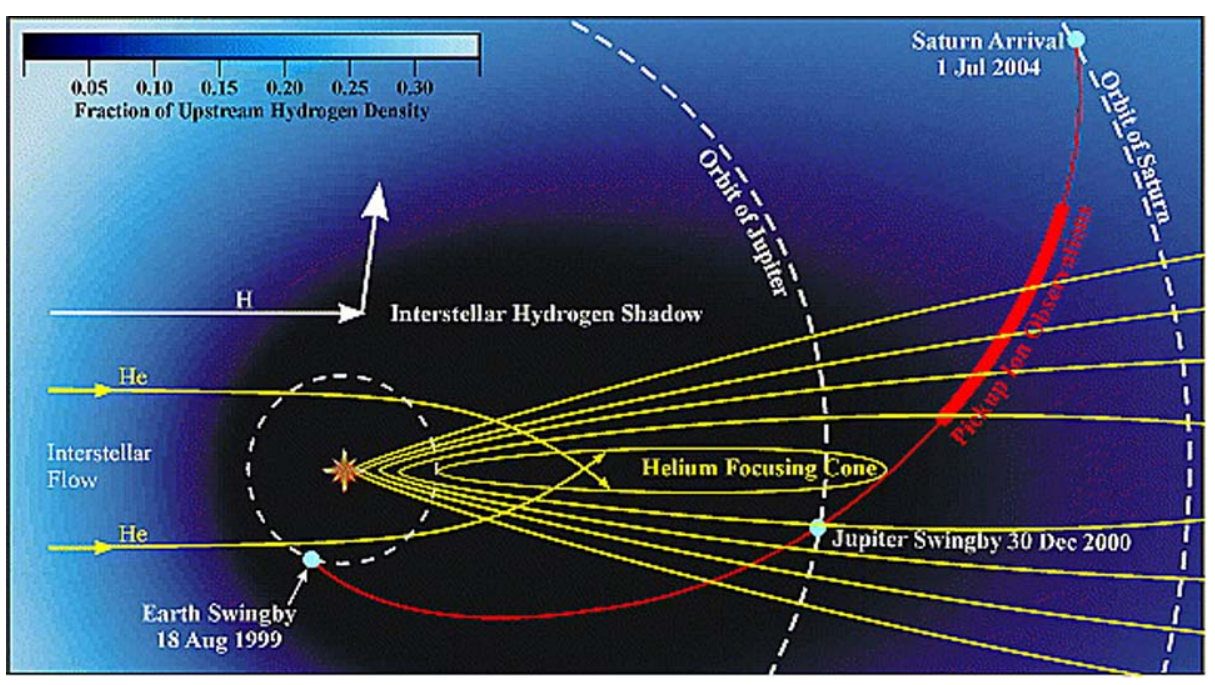

Fig. 3 Schematic diagram of the He focusing cone and the region of the Cassini trajectory between 6 and $8 \mathrm{AU}$ where observations of PUIs were made. The interstellar hydrogen shadow, caused by radiation pressure on $\mathrm{H}^{\circ}$ and photoionization, is also shown. (The figure is from McComas et al. 2004b)

interstellar $\mathrm{He}^{\mathrm{o}}$ at the termination shock. Since $\mathrm{He}^{\mathrm{o}}$ passes almost unimpeded through the outer heliosphere, these data give a CHISM velocity of $-26.3 \pm 0.5 \mathrm{~km} \mathrm{~s}^{-1}$, a temperature of $6306 \pm 390 \mathrm{~K}$, and $\mathrm{He}^{\mathrm{o}}$ density $n\left(\mathrm{He}^{\circ}\right)=0.0148 \pm 0.0020 \mathrm{~cm}^{-3}$ (Möbius et al. 2004). The downwind direction for interstellar $\mathrm{He}^{\circ}$ determined from Ulysses data and given in B1950 coordinates by Witte (2004) must then be corrected to J2000 coordinates for most purposes, yielding the direction $\lambda=75.4^{\circ} \pm 0.5^{\circ}$ and $\beta=-5.1^{\circ} \pm 0.2^{\circ}$ ( $\mathrm{J} 2000$, Witte, private communication, IBEX memo of August 6 2007). The early observations of the flow of interstellar $\mathrm{H}^{\circ}$ and $\mathrm{He}^{\circ}$ through the heliosphere found that the upwind directions of interstellar $\mathrm{H}^{\circ}$ and $\mathrm{He}^{\circ}$ directions are offset by $\sim 15^{\circ}$ (Weller and Meier 1974). The more precise $\mathrm{He}^{\circ}$ and $\mathrm{H}^{\circ}$ (Lallement et al. 2005) data now available indicate an offset angle of $\sim 4.9^{\circ} \pm 1.0^{\circ}$ (Frisch 2007, 2008a).

The CHISM parameters traced by $\mathrm{He}^{\mathrm{o}}$ define important details of the heliosphere interaction with the ISMa. For example, the sonic velocity for a perfect gas with solar composition at $\sim 6300 \mathrm{~K}$ is $\sim 8.2 \mathrm{~km} \mathrm{~s}^{-1}$, while the Alfven velocity for a medium with $n(\mathrm{e})=0.06 \mathrm{~cm}^{-3}$ and a magnetic field strength of $2.7 \mu \mathrm{G}$ is $24.0 \mathrm{~km} \mathrm{~s}^{-1}$ (Sects. 2.1.2, 4.3, 3.4, Spitzer 1978). The relative motion between the ISMa and the Sun as indicated by the $\mathrm{He}^{\mathrm{o}}$ data $\left(26.3 \mathrm{~km} \mathrm{~s}^{-1}\right)$ indicates that the heliosphere bow shock would be supersonic with Mach number $M_{\mathrm{C}} \sim 3$, if only acoustic velocities are considered. If Alfvenic wave propagation is also considered, the interaction would be barely super-magnetosonic with $M_{\mathrm{A}} \sim 1$ if the ISMF direction is approximately perpendicular to the shock normal (e.g. a perpendicular shock).

\subsubsection{Hydrogen}

Contrary to helium, neutral interstellar hydrogen is strongly coupled to protons in the interstellar gas by resonant charge exchange and thus is modified within the heliospheric interface. The basic understanding of the related heliosphere-LIC interaction has been summarized in early reviews (Axford 1972; Fahr 1974; Holzer 1989; Thomas 1978), as well as con- 
temporary discussions (Fahr 1991; Baranov and Malama 1993; Ruciński et al. 1993; Zank et al. 1996; Izmodenov et al. 1999a, 2004; Fahr et al. 2000; Müller et al. 2000; Heerikhuisen et al. 2006).

On meeting the obstacle created by the expanding solar wind, the (most probably) supersonic interstellar plasma adapts to the changed flow conditions by decelerating through the heliospheric bow shock to a subsonic speed, and flowing along the heliopause. At the same time, the expanding solar wind plasma becomes compressed and heated up at the termination shock (TS), where $\sim 75 \%$ of the energy transfer goes into the pickup ion population (Richardson et al. 2008). Since the charge-exchange coupling length between the protons and neutral atoms in the CHISM is up to a few hundred AU, depending on proximity to the heliopause, i.e. on the order of the size of the entire heliosphere, the neutral gas does not adapt immediately to the changed flow conditions, and $\geq 50 \%$ of the neutral gas becomes kinematically decoupled from the charged component of interstellar gas.

The charge-exchange interaction does not stop, however, and since the charge exchange does not involve significant momentum exchange between the individual colliding particles, two new populations of particles between the bow shock and the heliopause are created. One of them consists of the former neutral atoms which lost their electrons due to charge exchange and became protons. Since they are subjected to electromagnetic interactions with the locally-thermalized ambient plasma, they quickly become incorporated and thermalized. At the same time, they are abundant enough to significantly affect the local thermodynamic parameters of the plasma, which becomes denser, faster, and cooler than in the absence of these new particles. The net pressure exerted on the plasma due to the charge exchange pushes the heliopause closer towards the Sun. Other important factors influencing the heliosphere configuration include the ISMF and the solar wind (e.g. Pogorelov et al. 2007; Opher et al. 2008).

The other charge-exchange products are the former protons from the outer heliosheath that acquired electrons from the flow of interstellar $\mathrm{H}^{\circ}$ atoms and became decoupled from the local electromagnetic environment, while maintaining the velocities they had at the moment of interaction. Thus a new population of neutral atoms is created, the so-called secondary population. Since these neutral atoms inherit the local kinematic parameters of the plasma, they are much warmer than the primary interstellar atoms, but their bulk velocity is much lower than the velocity of the primary population. Since they are not tied to the ambient magnetic field, they can travel freely at large distances from their birthplaces without a change in their kinematic parameters. As a result, the local temperature and bulk velocity of the neutral component are different from the local temperature and bulk velocity of the ambient plasma. In particular, the secondary population becomes overdense, heated up, and slowed down as compared with the primary population. Thus, it forms a structure referred to as the "hydrogen wall", or alternatively as the "Baranov wall" (Baranov et al. 1991). The signature of this feature on interstellar Ly $\alpha$ absorption features is seen towards many stars (Sect. 3.2).

The original population of interstellar atoms is attenuated during the passage through the outer heliosheath, and since the charge-exchange process is more effective for the slow wing of this population, the primary population is accelerated by a few $\mathrm{km} \mathrm{s}^{-1}$ and cooled by a few hundred K. Thus the velocity of the primary population at the nose of the TS will be greater than the bulk velocity of interstellar helium, and the temperature will be a little lower than the helium temperature. Details depend on the parameters of the LIC.

Contrary to interstellar protons, neutral atoms from both the primary interstellar population and the secondary population created by charge exchange in the outer heliosheath penetrate freely inside the heliopause, to the inner heliosheath region, where the solar wind 
is heated up and decelerated to a subsonic speed by transition through the TS. The solar wind density decreases as $\sim R^{-2}$ inside of the TS, where $R$ is the distance to the Sun, so that the plasma density in this region is on the order of $10^{-4} \mathrm{~cm}^{-3}$ and lower by at least 3 orders of magnitude than the plasma density in the hydrogen wall. Thus the efficiency of charge exchange is reduced dramatically as compared with the efficiency in front of the heliopause, and the two populations flow through the inner heliosheath practically without a change. The few charge-exchange events occurring in this region result in the creation of another population of heliospheric neutral atoms, whose energy is equal to the typical energy of protons in the outer heliosheath, of about $0.5 \mathrm{keV}$. The distribution function of this population is strongly non-Maxwellian (Baranov et al. 1998; Izmodenov 2001; Izmodenov et al. 2001). This population, however tenuous it is, is important for the reasons of diagnostics of both heliospheric heliosheath and of the inner sheaths of astrospheres, because it can be observed spectroscopically (Linsky and Wood 1996; Wood et al. 2007). It is also the main target of observations by IBEX (McComas et al. 2004a, 2005, 2006). The atoms created by charge exchange inherit the local parameters of the plasma; owing to their relatively large speed they are able to penetrate close to the Sun with few losses, carrying the information on the conditions in their birth places.

The two thermal populations of neutral interstellar hydrogen at the nose of the TS, the primary and secondary, can be approximated (admittedly, less than perfect) by two Maxwellian functions shifted in velocity space (e.g. Baranov et al. 1998; Izmodenov 2000; Quémerais and Izmodenov 2002). Recent state-of-the-art models of the heliosphere tend to agree (Müller et al. 2008) that the combined density of the primary and secondary populations at this point is approximately two-fold lower than the neutral gas density in the CHISM (i.e., that the filtration factor of the hydrogen gas is equal to about 50\%). The breakdown between the primary and secondary populations and exact values of their densities, bulk velocities, and temperatures depend on the conditions in the unperturbed CHISM. For example, in a recent report on the determination of the density of neutral interstellar hydrogen at the TS, Bzowski et al. (2008) adopted the following parameters in the CHISM: the upwind direction $\lambda_{\mathrm{B}}=254.68^{\circ}, \phi_{\mathrm{B}}=5.31^{\circ}$ in the B1950.0 ecliptic coordinates (Witte 2004; Möbius et al. 2004); bulk velocity $V_{\mathrm{B}}=26.3 \mathrm{~km} \mathrm{~s}^{-1}$; temperature $T_{\mathrm{B}}=6400 \mathrm{~K}$; density of neutral He equal to $0.015 \mathrm{~cm}^{-3}$; and based on the He ionization degree in the LIC inferred by Wolff et al. (1999) on the level of $\sim 30-40 \%$, the density of $\mathrm{He}^{+}$equal to $0.008 \mathrm{~cm}^{-3}$, in agreement with He ionization levels predicted by radiative transfer models of the CHISM (Sect. 4.3). With these parameters, they additionally modeled two cases of proton and $\mathrm{H}$ atom densities in the CHISM, and obtained the following modifications of parameters of the primary and secondary populations at the nose of the TS:

1. Input CHISM values: proton density $n_{\mathrm{p}}=0.06 \mathrm{~cm}^{-3}$; neutral gas density $n_{\mathrm{H}}=$ $0.18 \mathrm{~cm}^{-3}$.

- TS Primary:

$n_{\mathrm{TS}, \text { pri }}=0.19 n_{\mathrm{H}, \mathrm{pri}}=0.035 \mathrm{~cm}^{-3} ; V_{\mathrm{TS}, \text { pri }}=1.08 V_{\mathrm{B}}=28.5 \mathrm{~km} \mathrm{~s}^{-1} ; T_{\mathrm{TS}, \text { pri }}=6020 \mathrm{~K}$

- TS Secondary:

$n_{\mathrm{TS}, \mathrm{sec}}=0.33 n_{\mathrm{H}}=0.060 \mathrm{~cm}^{-3} ; V_{\mathrm{TS}, \mathrm{sec}}=0.71 V_{\mathrm{B}}=18.7 \mathrm{~km} \mathrm{~s}^{-1} ; T_{\mathrm{TS}, \mathrm{sec}}=16300 \mathrm{~K}$

- Hence the resulting net density of interstellar $\mathrm{H}$ at the TS:

$n_{\mathrm{H}, \mathrm{TS}}=0.53 n_{\mathrm{H}}=0.095 \mathrm{~cm}^{-3}$.

2. Input CHISM values: proton density $n_{\mathrm{p}}=0.032 \mathrm{~cm}^{-3}$; neutral gas density $n_{\mathrm{H}}=$ $0.2 \mathrm{~cm}^{-3}$.

- TS Primary:

$n_{\mathrm{TS}, \text { pri }}=0.29 n_{\mathrm{H}}=0.059 \mathrm{~cm}^{-3} ; V_{\mathrm{TS}, \text { pri }}=1.07 V_{\mathrm{B}}=28.2 \mathrm{~km} \mathrm{~s}^{-1} ; T_{\mathrm{TS}, \text { pri }}=6100 \mathrm{~K}$ 
- TS Secondary:

$n_{\mathrm{TS}, \mathrm{sec}}=0.29 n_{\mathrm{H}}=0.059 \mathrm{~cm}^{-3}, V_{\mathrm{TS}, \mathrm{sec}}=0.70 V_{\mathrm{B}}=18.5 \mathrm{~km} \mathrm{~s}^{-1}, T_{\mathrm{TS}, \mathrm{sec}}=16500 \mathrm{~K}$

- Hence the resulting net density of interstellar $\mathrm{H}$ at the TS:

$n_{\mathrm{H}, \mathrm{TS}}=0.59 n_{\mathrm{H}}=0.117 \mathrm{~cm}^{-3}$.

The CHISM input parameters for Case 1 above give the best match to the CHISM properties predicted by radiative transfer models (Sect. 4.3).

Inside the TS, the atoms can be treated as collisionless. They propagate under the influence of the joint action of solar gravity and solar radiation pressure, and they suffer losses due to ionization by solar wind and solar EUV radiation. Ionization results in creation of pickup ions (Sect. 2.2) in the solar wind and of another population of neutral atoms, the socalled Neutral Solar Wind, which is composed of solar wind protons that acquired electrons from the incoming interstellar gas and propagate away from the Sun while maintaining the original kinematic parameters of the parent protons.

The radiation pressure generally (over)compensates solar gravity. Its strength depends on the net intensity in the solar Ly $\alpha$ line and-since the line features a non-flat, self-reversed shape - on the instantaneous radial velocity of the atom. Since the solar EUV output varies with time on timescales from weeks (solar rotation period) to decades (the solar cycle period), the effective compensation of solar gravity varies from a factor of 0.9 at solar minimum to a factor of 1.6 at solar maximum (Bzowski 2001), the trajectories of the atoms are not Keplerian, and both density and bulk velocity of the atoms change with time (Ruciński and Bzowski 1995; Bzowski et al. 1997). Another factor modifying the local density and bulk velocity of the atomic ensemble is ionization, which differentiates the members of the ensemble by energy, yielding an apparent acceleration of the flow by several $\mathrm{km} \mathrm{s}^{-1}$ within a few AU from the Sun, as well as a deflection of the local flow direction relative to the original interstellar flow vector. Ionization also varies on time scales from days to decades and additionally features a latitudinal anisotropy that depends on solar cycle phase and is especially pronounced during solar minimum period. The attenuation of density at the upwind axis within a few AU from the Sun is approximately exponential.

The photons of the solar $\mathrm{Ly} \alpha$ radiation are scattered off the neutral $\mathrm{H}$ atoms and produce the heliospheric glow. The maximum of the signal when observed from 1 AU originates at a few AU (typically, when looking upwind, from 2-5 AU, depending on the phase of solar cycle), i.e. from the region where the distribution function of the gas is quite complex.

The net density of the gas at a point $R$ on the inflow axis is composed of the densities of the two collisionless components, the primary and secondary atoms, that have different bulk velocities and temperatures, and which additionally vary with solar cycle phase and distance from the Sun. Their thermal spread along the inflow axis decreases between $\sim 10 \mathrm{AU}$ and $1 \mathrm{AU}$ from the original values at the TS, determined by the temperatures; the decrease is $3 \mathrm{~km} \mathrm{~s}^{-1}$ at solar minimum or $2 \mathrm{~km} \mathrm{~s}^{-1}$ at solar maximum. Simultaneously, the bulk velocity of the two components increases by 4 to $6 \mathrm{~km} \mathrm{~s}^{-1}$ except for the primary population during solar maximum, which is slowed down by $2 \mathrm{~km} \mathrm{~s}^{-1}$. This is illustrated in Fig. 4. Both populations show appreciable radial velocity gradients at the region of space where most of the spectral line of the heliospheric Ly $\alpha$ glow is created, which also varies during the solar cycle. In addition, the densities of these populations also feature a strong gradient, illustrated in Fig. 5. The Ly $\alpha$ backscattered line created by the primary and secondary $\mathrm{H}^{\circ}$ populations during solar minimum conditions is shown in Quémerais and Izmodenov (2002), and the expected profile as a function of solar cycle phase is given in Quémerais et al. (2006). The difference in bulk velocities of the two populations in the region where the 
Radial velocity upwind

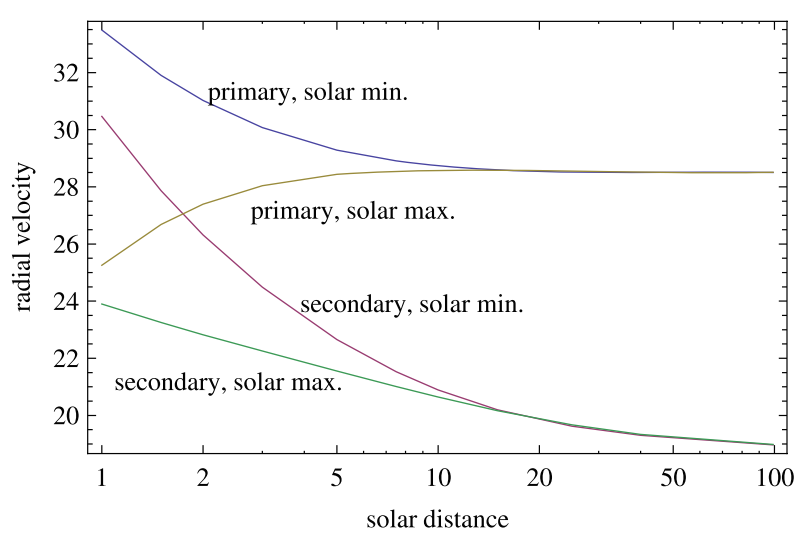

Fig. 4 Bulk velocity of the primary and secondary populations of neutral interstellar $\mathrm{H}$ for solar minimum and maximum conditions along the inflow axis, starting with initial conditions at the termination shock (figure right). Units of the horizontal axis are $\mathrm{AU}$, the units of the vertical axis are $\mathrm{km} \mathrm{s}^{-1}$. The profiles were calculated using the Warsaw time-dependent kinetic model of heliospheric hydrogen (Bzowski et al. 1997) with time-dependent radiation pressure and ionization rate, and assuming the boundary conditions at the termination shock according to case 2, which seem to be close to actual parameters in this region based on analysis of Ulysses pickup ion measurements by Bzowski et al. (2008). The radiation pressure for 1996.0, adopted as the solar minimum case, was equal to $\mu=0.9$; for 1999.5, adopted as the solar maximum case, it was equal to $\mu=1.4$

Fig. 5 Density of the primary and secondary populations of neutral interstellar $\mathrm{H}$ for solar minimum and maximum conditions along the inflow axis. The horizontal axis is scaled in $\mathrm{AU}$, the vertical axis in $\mathrm{cm}^{-3}$. The profiles were calculated using identical assumptions as in the case of velocities shown in Fig. 4

\section{Density of neutral $\mathrm{H}$ upwind}

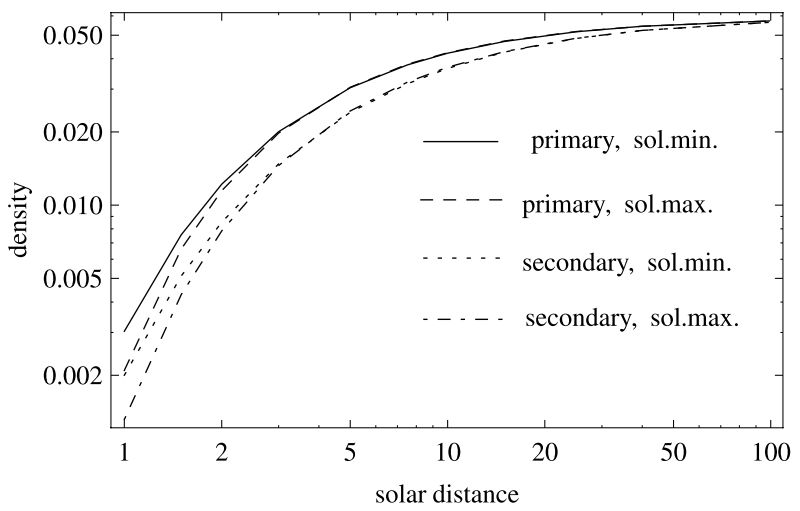

backscattered Ly $\alpha$ line is formed is approximately equal to half of the thermal spread. Thus the two populations overlap in the velocity space and create a spectral line with a single peak.

\subsection{Pickup Ions and Anomalous Cosmic Rays}

Cosmic ray data from the early 1970s reported particles at unexpected energies of $\sim 10 \mathrm{MeV} /$ nucleon, showing anomalous compositions with enhanced $\mathrm{He}, \mathrm{H}, \mathrm{O}, \mathrm{N}, \mathrm{Ne}$, and Ar abundances (e.g. Gloeckler and Wenzel 2001). Fisk et al. (1974) recognized that 
this population consisted of elements with high first ionization potentials (FIP) that were expected to be neutral in the ISMa. Neutral interstellar gas flows into the heliosphere, and is ionized through charge exchange with the solar wind (for interstellar $\mathrm{H}, \mathrm{N}, \mathrm{O}$, $\mathrm{Ne}$, and $\mathrm{Ar}$ ), photoionization (for interstellar $\mathrm{He}, \mathrm{H}$, and $\mathrm{Ne}$ ), or electron impact ionization (for interstellar He, N, and Ar, Ruciński et al. 1996; Cummings et al. 2002). The ionized interstellar atoms are picked up by the solar wind and convected outwards to the outer heliosphere, forming a suprathermal ion population known as pickup ions (PUIs). The PUIs then become accelerated to form the anomalous cosmic rays (ACRs, Sect. 3.1). Pickup He was discovered by Möbius et al. (1985). Pickup ions of $\mathrm{H},{ }^{4} \mathrm{He}$ and ${ }^{3} \mathrm{He}, \mathrm{N}, \mathrm{O}$, Ar, and Ne have now been measured in the inner 5 AU by several different spacecraft, including Ulysses data obtained at all ecliptic latitudes (Möbius et al. 1985; Gloeckler and Fisk 2007; Gloeckler et al. 2004; Gloeckler and Geiss 2004). The observed PUI population consists of ions with an interstellar origin, that are mixed with an inner source of PUIs that peaks near the Sun and consists of low-FIP elements (Geiss et al. 1995; Schwadron and Gloeckler 2007). The inner source ions appear to originate from the recycling of solar wind on dust grains in the inner heliosphere, and thereby trace the solar composition. The unique signature of PUIs with an interstellar origin is the energy cutoff at twice the solar wind velocity, caused as the PUI energies are isotropized in the solar wind rest frame (e.g. Gloeckler and Fisk 2007).

The low charge exchange and similar photoionization cross sections of $\mathrm{He}$ and $\mathrm{Ne}(\mathrm{Ru}-$ ciński et al. 1996) indicate that both interstellar elements reach the inner heliosphere as neutrals, and are gravitationally focused downwind of the Sun into the He focusing cone (Fig. 3, Möbius et al. 2004; McComas et al. 2004b). The He focusing cone is seen in $584 \AA$ backscattered emission, as well as enhanced densities of He PUIs (Möbius et al. 2004; Gloeckler et al. 2004). Since the He focusing cone is formed by interstellar $\mathrm{He}^{\mathrm{o}}$ atoms arriving from all ecliptic latitudes, and ionization rates vary with ecliptic latitude $\beta$, solar activity proxies are required to reconstruct the $\mathrm{He}^{\mathrm{o}}$ ionization rate from the EUV radiation field over the full solar surface. The phase space density of the PUIs is proportional to the product of the local neutral density and the ionization rate, and can be used to estimate the maximum density of $\mathrm{He}^{\mathrm{o}}$ in the focusing cone. The $\mathrm{He}^{\mathrm{o}}$ density in the focusing cone varies over the solar cycle, from $\sim 0.01 \mathrm{~cm}^{-3}$ during solar maximum to $\sim 0.045 \mathrm{~cm}^{-3}$ during solar minimum, compared to the interstellar value of $0.015 \mathrm{~cm}^{-3}$ (Sect. 2.1.1). The higher focusing cone density during solar minimum conditions follows because the ionization rate varies approximately by a factor of 3 over the solar cycle, with higher rates during solar maximum because of increased solar EUV emission. During solar minimum, the interstellar upwind He density at $1 \mathrm{AU}$ is $80 \%$ to $85 \%$ of the CHISM density $\left(\sim 0.015 \mathrm{~cm}^{-3}\right)$, while during solar maximum it is $\approx e^{-1}$ of the CHISM He density $\left(\sim 0.005 \mathrm{~cm}^{-3}\right)$. The result is that the product of density and ionization rate at $1 \mathrm{AU}$ is approximately constant over the solar cycle to within $20-25 \%$ in the upwind direction. In the peak of the cone the He PUI phase space density is between a factor 2 (solar maximum) and a factor of almost 4 (solar minimum) higher than in the upwind direction defined by the interstellar flow.

In situ data from Cassini found $\mathrm{H}, \mathrm{He}$, and O PUIs out to distances of 6 to $8 \mathrm{AU}$ downwind of the Sun (McComas et al. 2004b). The He data showed that the focusing cone extends beyond 8 AU downwind. The higher He PUI density in the focusing cone was associated with a decrease in H PUIs in the downwind hydrogen shadow caused by radiation pressure on the inflowing interstellar $\mathrm{H}$. These in situ observations of the hydrogen shadow confirm the asymmetric distribution of $\mathrm{H}^{\circ}$ in the inner heliosphere inferred from remote observations of the interplanetary Ly $\alpha$ glow (Sect. 2.1.2). The Cassini trajectory and a simulation of the He focusing cone are shown in Fig. 3. 
The $\mathrm{H}, \mathrm{He}, \mathrm{O}, \mathrm{N}, \mathrm{Ne}$, and Ar PUI data provide valuable constraints on the neutrality as well as the chemical composition of the CHISM (Sect. 4.3). At low energies, $\leq 20 \mathrm{MeV} /$ nucleon, the isotopic composition of PUIs and ACRs show abundance ratios of ${ }^{15} \mathrm{~N} /{ }^{14} \mathrm{~N},{ }^{18} \mathrm{O} /{ }^{16} \mathrm{O}$, and ${ }^{22} \mathrm{Ne} /{ }^{20} \mathrm{Ne}$, that are near the solar values of $\sim 0.004,0.002$, and 0.07 , respectively (Gloeckler and Wenzel 2001; Cummings and Stone 2007). Helium isotopes show ${ }^{4} \mathrm{He} /{ }^{3} \mathrm{He} \sim 1.7 \times 10^{-4}$, which is similar to meteoritic and HII region values (Salerno et al. 2003; Gloeckler and Fisk 2007). These data suggest the CHISM composition is solar, independent of uncertainties in actual solar abundances.

\subsection{Large Interstellar Dust Grains in the Heliosphere}

In the early 1990s, after its Jupiter flyby, the Ulysses spacecraft positively identified interstellar dust in the solar system penetrating deep into the solar system. The Ulysses spacecraft detected impacts predominantly from a direction that was opposite to the expected impact direction of interplanetary dust grains. It was found that, on average, the impact velocities exceeded the local solar system escape velocity (Grün et al. 1994). The motion of the interstellar grains through the solar system was parallel to the flow of neutral interstellar hydrogen and helium gas, both traveling at a speed of $26 \mathrm{~km} \mathrm{~s}^{-1}$ (Baguhl et al. 1995). The interstellar dust flow persisted at higher latitudes above the ecliptic plane, even over the poles of the Sun, whereas interplanetary dust is strongly depleted away from the ecliptic plane (Grün et al. 1997).

Ulysses has measured the interstellar dust stream at high ecliptic latitudes between 3 and 5 AU. Interstellar grains have also been observed with the in-situ dust detectors on board Cassini, Galileo and Helios (Altobelli et al. 2003, 2005, 2006), covering a heliocentric distance range between 0.3 and $3 \mathrm{AU}$ in the ecliptic plane. The masses of clearly identified interstellar grains range from $10^{-18} \mathrm{~kg}$ to about $10^{-13} \mathrm{~kg}$ with a maximum at about $10^{-15} \mathrm{~kg}$ (Landgraf et al. 2000). In Fig. 6 the total interstellar dust mass distribution of 896 ISDGs observed by Ulysses, to date, is plotted, and compared to the power law size distribution of grain radii, $n(a) \sim a^{-3.5}$, that provides a nominal match to extinction data given abundance constraints (Mathis et al. 1977, e.g. MRN distribution). Ulysses has recorded significant differences in the particle sizes at different heliocentric distances. The measurements reveal a

Fig. 6 The mass distribution of 896 interstellar dust grains detected by the Ulysses spacecraft inside the heliosphere (crosses) is shown as a function of grain mass. The corresponding grain radius is shown for the assumption of spherical silicate grains of density $2.5 \mathrm{~g} \mathrm{~cm}^{-3}$ (top axis). For comparison, an MRN interstellar dust grain distribution (Mathis et al. 1977) for an assumed total interstellar mass density of $0.25 \mathrm{~cm}^{-3}$ hydrogen atoms in the CHISM is plotted (dashed line)

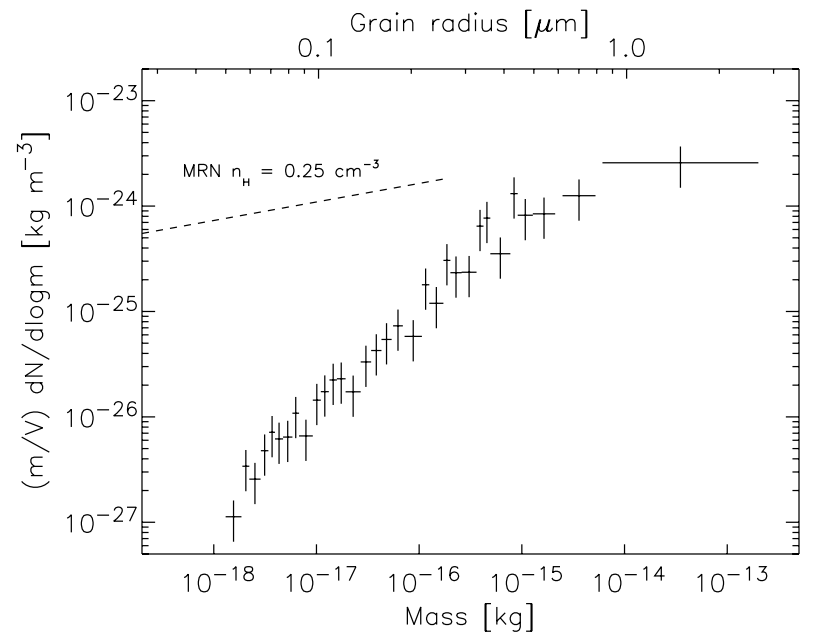


lack of small $\left(<3 \times 10^{-16} \mathrm{~kg}\right)$ interstellar grains inside $3 \mathrm{AU}$ heliocentric distance (Landgraf et al. 2000). Measurements by Cassini and Galileo in the distance range between 0.7 and $3 \mathrm{AU}$ showed that interstellar particles are bigger than $10^{-15} \mathrm{~kg}$, with increasing masses closer to the Sun (Altobelli et al. 2003, 2005). The trend of increasing particle masses close to the Sun was also seen by Helios, which recorded particles of ca. $10^{-14} \mathrm{~kg}$ mass down to $0.3 \mathrm{AU}$ (Altobelli et al. 2006). These data are consistent with an interstellar dust stream filtered by solar radiation pressure. Interstellar particles with optical properties of astronomical silicates or organic refractory materials are consistent with the observed radiation pressure effects (Landgraf et al. 1999), however the overabundance of carbon in the CHISM (Sect. 4.3) appears to rule out a significant proportion of organics. The heliospheric trajectories of the largest ISDGs with radii $\geq 7 \mu \mathrm{m}$ are determined by solar gravity. These large grains are predicted to form a "focusing cone" downwind of the Sun (Grogan et al. 1996; Landgraf 2000).

Ulysses has monitored the interstellar dust flow through the solar system for more than 16 years. This time period covers more than two and a half revolutions of the spacecraft about the Sun through more than $2 / 3$ of a complete 22 -year solar cycle. Thus, Ulysses measured interstellar dust during solar minimum and solar maximum conditions of the interplanetary magnetic field (IMF). In mid 1996, a decrease by a factor 3 of the interstellar dust flux density from initial values of $1.5 \times 10^{-4} \mathrm{~m}^{-2} \mathrm{~s}^{-1}$ was observed. This decrease was attributed to the increased filtering of small grains by the solar wind magnetic field during solar minimum conditions (Landgraf 2000). Since early 2000, Ulysses has detected interstellar dust flux levels above $10^{-4} \mathrm{~m}^{-2} \mathrm{~s}^{-1}$ again (Landgraf et al. 2003). Until early 2005 the upwind direction of the interstellar grains was within a few tens of degrees in agreement with the interstellar helium flow direction (Landgraf and Grün 1998; Frisch et al. 1999; Krüger et al. 2006). During 2000 and 2006 Ulysses was traveling through almost the same spatial regions and with almost identical detection geometries for interstellar grains; however the upwind direction of the grains was somewhat wider and shifted by about 30 degrees away from the helium flow direction towards southern ecliptic latitudes during the latter set of observations (Krüger et al. 2007).

The flux variations and the deficiency of small grain masses $\left(<10^{-16} \mathrm{~kg}\right)$ indicate a time dependent depletion of small interstellar grains inside the heliosphere. Several groups have studied the alteration of the interstellar dust flux inside the heliosphere by solar radiation pressure force, gravitational focusing and the interactions with the time varying interplanetary magnetic field (Grogan et al. 1996; Landgraf et al. 1999; Landgraf 2000, 2003; Mann and Kimura 2000; Czechowski and Mann 2003b; Linde and Gombosi 2000).

\section{The Edges of the Heliosphere: Interstellar Material and the Interstellar Magnetic Field}

The outermost regions of the heliosphere that are closest to the ISMa are the least explored, but show visible evidence of the interaction between the ISMa and heliosphere. The diverse evidence for ISMa in the outer heliosphere regions include anomalous cosmic rays, decelerated interstellar hydrogen in the hydrogen wall, and possibly small excluded interstellar dust grains signaled by their absence inside of the heliosphere and polarization of light from nearby stars.

\subsection{Anomalous Cosmic Rays and Heliospheric Asymmetries}

Observations show that V2 crossed the TS much closer to the Sun ( $~ 84$ AU, Richardson et al. 2008) than V1 ( 94 AU, Stone et al. 2005). While the heliospheric boundaries are 
Fig. 7 Schematic diagram of an equatorial cut through the termination shock taken from McComas and Schwadron (2006). The Archimedean spiral flux tubes first cross the termination shock near the nose and their connection points move back along the flanks of the shock as they continue to propagate out through the heliosheath. The long connection times for the flanks and tail can allow diffusive shock acceleration to energize particles up to observed ACR energies

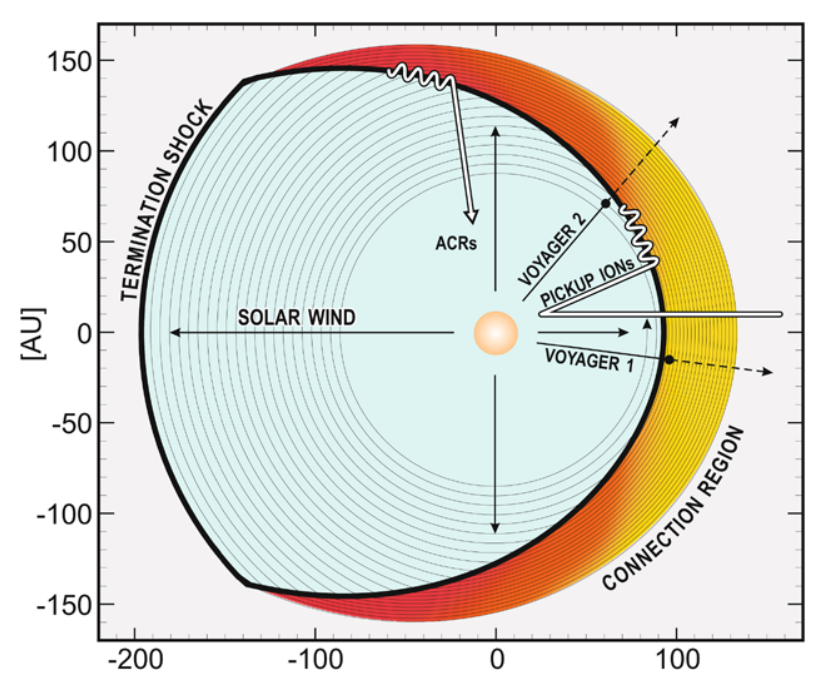

not time stationary, this large a difference almost certainly reflects at least some consistent north-south asymmetry of the TS. The ISMF at the heliosphere (Sect. 3.4) likely produces significant asymmetrical distortions of the TS and heliopause (HP, Linde et al. 1998; Ratkiewicz et al. 1998; Pogorelov and Matsuda 1998; Pogorelov et al. 2004) and is the most likely cause of this observed asymmetry.

Magnetohydrodynamic (MHD) models of the heliosphere that include an ISMF help quantify its effects on the global heliosphere. Opher et al. (2007) showed that a field tilted $60^{\circ}$ from the galactic plane matches both the flow data and the radio emission data. This tilt creates a large north-south and some east-west asymmetries. This model also predicts that the distance of the TS in the direction of V2 is 8-11 AU closer than in the direction of V1, consistent with actual crossing distances. The same model further suggests that the HP distance may be 13-24 AU closer in the direction of V2. Pogorelov et al. (2008b) argue that the strength of the interstellar field can be increased to strengths of $\sim 4 \mu \mathrm{G}$ to reproduce the heliospheric asymmetry seen by the V1 and V2 (also see Pogorelov et al. 2007). However, once the effects of neutrals are included, these asymmetries become much smaller and no longer match the large difference seen in TS crossing distances between V1 and V2 (Pogorelov et al. 2006; Heerikhuisen et al. 2008). An additional complication in the interpretation of the heliosphere asymmetry seen by V1 and V2 is that the solar wind dynamic pressure decreased significantly between the two crossings.

MHD models also reveal strong nose-to-tail asymmetries in the TS geometry (Zank 1999), as shown in Fig. 7. McComas and Schwadron (2006) showed that solar wind magnetic field lines will first make contact at the nose of the TS, because the location of the nose is much closer to the Sun than anywhere else along the shock. Diffusive acceleration of ions in the compressed magnetic field beyond the TS requires large amounts of time, typically on the order of a year, to achieve Anomalous Cosmic Ray (ACR) energies. Over this large timescale, the connection point between solar wind field lines and the TS moves from the nose to the flanks and tail. ACR acceleration initiates in this connection region. Therefore, the ACR energies should reach their highest intensities back on the flanks and tail of the TS. Thus, the absence of enhanced of ACR fluxes noted by V1 and V2 in the TS regions can be explained naturally by the large nose-to-tail asymmetry of the TS. Schwadron and McComas (2007) showed that this asymmetry also likely explains the strong correlation between 
ACRs and galactic cosmic rays observed in the heliosheath. Finally, Schwadron et al. (2008) provided the first detailed analytic model of ACRs accelerated along a non-symmetric TS, and showed good agreement between results of the model and ACR observations by V1.

Anomalous cosmic rays are formed by the acceleration of PUIs, which in turn are interstellar atoms with high first ionization potentials, that survived passage through the heliosheath regions. ACRs provide an additional measurement of the ISMa, after correcting for ionization, acceleration and propagation effects. Measurements by the Voyager spacecraft show $\mathrm{He}, \mathrm{O}, \mathrm{N}, \mathrm{Ar}$, and Ne ACRs in the outer heliosphere, with abundances that are consistent with abundances of the parent neutrals in the CHISM (e.g. Cummings et al. 2002). Observations of $\mathrm{He}, \mathrm{N}, \mathrm{O}$, and $\mathrm{Ne}$ isotopes in the ACR population show that the composition of the CHISM is approximately solar (Sect. 2.2, Cummings and Stone 2007), providing valuable insights into the overall ISMa abundances.

\subsection{Decelerated Interstellar $\mathrm{H}^{\circ}$ in the Outer Heliosheath Region}

Interstellar material in the outer heliosphere can be studied directly using ultraviolet (UV) Ly $\alpha$ spectra of nearby stars taken by the HST. As it enters the heliosphere, neutral H from the CHISM is heated and decelerated through charge exchange processes in the hydrogen wall (Sect. 2.1.2). This heliospheric hydrogen produces a Ly $\alpha$ absorption signature that has been detected in some HST spectra (Linsky and Wood 1996; Wood et al. 2005). The heliospheric contribution to the hydrogen wall can be completely obscured by the interstellar absorption if the ISMa column density is too high and the ISMa absorption therefore too broad.

There are actually two distinct populations of interstellar hydrogen that are detectable. The dominant population consists of neutrals formed by charge exchange in the hydrogen wall between the heliopause and bow shock (Sect. 2.1.2). Most detections of heliospheric absorption sample this population, and originate near the upwind direction of the CHISM flow, because this is where the heating, compression, and deceleration of the ISMa neutrals is strongest. Deceleration of the $\mathrm{H}^{\circ}$ flow in the radial direction is required to separate the heliospheric absorption from that of the ISMa (Wood et al. 2005). Several studies have demonstrated that hydrodynamic models of the heliosphere can reproduce the observed kinematic characteristics of the hydrogen wall absorption, depending on exactly what is assumed about the properties of the CHISM (Gayley et al. 1997; Wood et al. 2000a, 2007; Izmodenov et al. 2002).

Besides the dominant hydrogen wall component, a second population of heliospheric hydrogen is a heliosheath component formed by charge exchange in the inner heliosheath between the heliopause and termination shock (Sect. 2.1.2). The very broad, weak Ly $\alpha$ absorption that this population produces is much harder to detect than the stronger hydrogen wall signature. In upwind and sidewind directions, the inner heliosheath is too narrow for there to be detectable heliosheath absorption; in downwind directions, line-of-sight distances through this region are longer and the absorption is detectable through its kinematic properties (Izmodenov et al. 1999b; Wood et al. 2007).

Although harder to detect and study, it is the heliosheath population of neutrals that is most relevant to IBEX, because most of the ENAs that IBEX will study are from this population rather than the hydrogen wall population. While IBEX will make detailed allsky maps of local ENA fluxes, and detailed measurements of their energy distributions from $\sim 10 \mathrm{eV}$ to $6 \mathrm{keV}$, the heliosheath absorption measurements provide information about lineof-sight integrated energy distributions, particularly in the $0.01-0.2 \mathrm{keV}$ energy range. Wood et al. (2007) have used constraints from the $\operatorname{Ly} \alpha$ data to quote a very conservative flux upper limit of $F_{0.01-0.2 \mathrm{keV}}<5000 \mathrm{~cm}^{-2} \mathrm{~s}^{-1} \mathrm{ster}^{-1}$ for the ENA flux that IBEX can expect to see from $0.01-0.2 \mathrm{keV}$ emissions. 
More precise constraints on ENA fluxes from the Ly $\alpha$ data (and future direct comparisons of IBEX and HST measurements) will require the assistance of kinetic heliospheric models that not only properly model the neutral velocity distributions throughout the heliosphere (Baranov and Malama 1993; Baranov and Malama 1995; Zank 1999; Izmodenov et al. 2005; Heerikhuisen et al. 2007; Pogorelov et al. 2007), but also extend at least 3000 AU downwind in order to be able to capture all the absorption in the downwind directions where the heliosheath absorption is detectable (Izmodenov and Alexashov 2003; Alexashov et al. 2004; Wood et al. 2008). These models should ideally use a sophisticated multicomponent treatment of the plasma in the heliosphere instead of the usual simple single-fluid treatment, as multicomponent models have been found to result in significantly different heliosheath neutral populations, and better reproduction of heliosheath absorption (Malama et al. 2006; Wood et al. 2007). In any case, once IBEX data are available it will be very interesting to see if models are capable of simultaneously reproducing both the local ENA spectra seen by IBEX and the Ly $\alpha$ absorption data.

\subsection{Small Excluded Interstellar Dust Grains}

The observed mass distribution of interstellar dust grains inside of the heliosphere is controlled by the original interstellar charge-to-mass ratios, $Q / m$, of incident ISDGs, graincharging in heliosheath regions, and the relative strengths of gravitational focusing, radiation pressure, and Lorentz forces inside of the heliosphere (Landgraf 2000). Interstellar grains with large $Q / m$ ratios are excluded from the heliosphere, as shown by the deficit of small grains in the Ulysses and Galileo data (Sect. 2.3, Fig. 6, Frisch et al. 1999) with radii $a \lesssim 0.2 \mu \mathrm{m}$ in comparison to the MRN power-law distribution of $n(a) \sim a^{-3.5}$ (Mathis et al. 1977). The very smallest carbonaceous grains, either graphites or polycyclicaromatic-hydrocarbons (PAH) with radii $<0.01 \mu \mathrm{m}$, appear to be missing from the CHISM entirely because all carbon is in the gas phase (Sect. 4.3, Slavin and Frisch 2008). The exclusion of small interstellar dust grains from the heliosphere has been discussed in many papers (Frisch et al. 1999; Czechowski and Mann 2003a; Mann and Kimura 2000; Grün et al. 2005).

The mass distribution of the excluded grains in principle can be obtained by comparisons between the observed grain mass distributions, and the 'true' interstellar mass distribution. Unfortunately, the ISDG mass distribution for very low-column-density ISMa such as the LIC has not been determined. LIC grains differ from grains in high-opacity clouds, in that they show high abundances of refractories in the gas due to grain destruction in shocks (Sect. 4.3, Frisch et al. 1999).

In contrast to the interstellar gas and small ISDGs that encounter the heliosphere, large ISDGs are not directly affected by the MHD boundaries. This is because the coupling of the grains to the gas is mediated primarily by the magnetic field, with only the relatively weak coupling of collisional and plasma drag acting directly on the grains. In the frame of reference of the heliosphere, the grains flow in with the interstellar gas and magnetic field at the relative speed of $\sim 26 \mathrm{~km} \mathrm{~s}^{-1}$. Once in the outer heliosheath, the most important force on the grains is the Lorentz force as the gas and (frozen-in) field is slowed and deflected at the nose of the heliopause.

The primary determinant of the dynamics of small grains in the outer heliosphere is Lorentz coupling to the magnetic field, which is mediated by the charge-to-mass ratio of grains, $Q / \mathrm{m}$. The charging rates for grains are very high, so we can assume that they are in equilibrium generally speaking. The primary sources of grain charging are the far UV radiation field, which charges the grains positively through the ejection of photoelectrons, and 


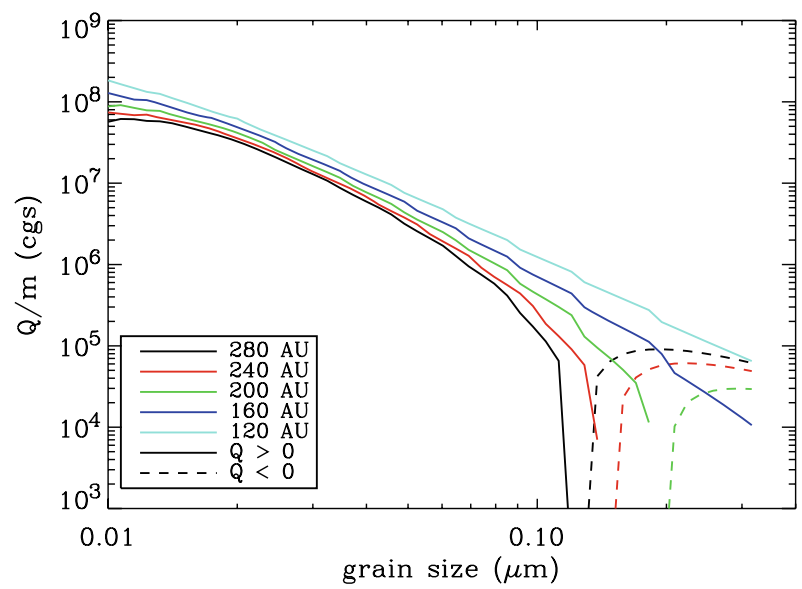

Fig. 8 Charge-to-mass ratio for silicate grains interacting with the heliosphere. As grains get closer to the Sun, solar UV radiation increases the charge of small grains through photoejection of electrons. The higher work function of larger grains impedes the photoejection of electrons, and larger collision rates of electrons drive the grain charge negative. Grains with positive and negative charges are denoted by solid and dashed lines, respectively. The distance of the grain from the Sun is denoted by the line color, with the most distant small grains having the least charge

the hitting and sticking of electrons on the grains. With these rates in balance, detailed calculations of the grain charging using rates and codes from Weingartner and Draine (2001), we find that the charge on a grain ranges from $Z=14$ to 137 for grain radii ranging from $a_{\mathrm{gr}}=0.01-0.3 \mu \mathrm{m}$ (Avinash et al. 2008). Grain charge increases as grains are exposed to higher fluxes of solar radiation (Fig. 8). The corresponding gyroradii depend on assumptions for the magnetic field and will vary as a function of position relative to the nose and within the heliosheath. For a $5 \mu \mathrm{G}$ field just inside the bow shock and a relative velocity of $14 \mathrm{~km} \mathrm{~s}^{-1}$ between the inflowing gas and dust, we find gyroradii of 0.7-2000 AU for this range in grain sizes. Thus small grains with their relatively small gyroradii will couple tightly to the field and be diverted along with the field and gas, while the largest grains will pass through the heliopause relatively unaffected (Sect. 2.3).

\subsection{Interstellar Magnetic Field at the Heliosphere}

The Faraday rotation of local and extragalactic radio point sources show that the global ISMF in the interarm region surrounding the Orion spur (Fig. 2) is directed clockwise around the galactic center when viewed from the north galactic pole (Han 2006). A major distortion of the ordered global magnetic field occurs in the solar vicinity. This distortion is from expanding shock fronts generated by supernova and massive star winds, associated with star formation in the Scorpius-Ophiuchus Association over the past $\sim 15$ Myrs. These shock fronts have displaced the global local field that is parallel to the galactic plane into a giant superbubble, and created the distinct Loop I feature that dominates the magnetic field in the northern hemisphere and at the Sun (Berkhuijsen 1971). Frisch (1981) concluded that the Loop I supernova remnant has expanded to the Sun, after comparing the kinetics of ISMa inside of the heliosphere and towards nearby stars, and evaluating refractory abundances indicative of dust grain destruction in shocks. Wolleben (2007) used polarizations traced by $1.4 \mathrm{GHz}$ radio continuum data to define the magnetic field direction in the 'S1' subshell of Loop I, and concluded that the Sun is located in the rim of S1. 
Four phenomena indicate the direction of the interstellar magnetic field (ISMF) at the heliosphere: (1) The offset angle between the upwind directions of $\mathrm{H}^{\circ}$ and $\mathrm{He}^{\mathrm{o}}$ flowing into the heliosphere (Lallement et al. 2005). (2) The polarization of optical light from stars located 6-35 pc from the Sun (Tinbergen 1982; Frisch 2007). (3) The magnetic S1 subshell of the Loop I supernova remnant (Wolleben 2007; Frisch 2008b). (4) The $3 \mathrm{kHz}$ emissions detected by the V1 and V2 spacecraft from beyond the heliopause (Gurnett et al. 2006). The first three of these phenomena can be characterized by a position angle. Position angles (PA) are a useful tool for describing a direction in the sky. By definition a PA is defined in the plane of the sky. The position angle $P A_{\mathrm{E}}$ in ecliptic coordinates is the angle between a vector and a great-circle meridian passing through the ecliptic north pole, and increasing to the east.

In this paragraph these four phenomena are summarized: (1) It is necessary to first convert the $\mathrm{He}^{\mathrm{o}}$ upwind direction to J2000 coordinates (this correction was inadvertently neglected in Lallement et al. 2005), in order to characterize the PA of the offset between the inflow directions of $\mathrm{He}^{\circ}$ and $\mathrm{H}^{\circ}$. With this correction, the offset angle between the upwind directions of $\mathrm{He}^{\circ}$ and $\mathrm{H}^{\circ}$ is $4.9^{\circ} \pm 1.0^{\circ}$, and the PA defined by these two directions in the ecliptic coordinate system is $-38^{\circ} \pm 12^{\circ}$ (Frisch 2007). (2) The magnetic field direction in the plane of the sky, and with respect to the ecliptic plane, is given by $90^{\circ}-P A_{\mathrm{E}}$ for magnetically aligned ISDGs. The polarization of nearby stars towards the heliosphere nose yields $P A_{\mathrm{E}}=-25^{\circ} \pm 9^{\circ}$. These two ISMF tracers then suggest that the ISMF is inclined to the ecliptic plane by $\sim 59^{\circ} \pm 5^{\circ}$, but do not provide any information on the angle between the cloud velocity and field direction. Regarding method (3) above, the ISMF direction near the Sun found from Faraday rotation data has large uncertainties because of the low densities. If the shell is assumed to be purely spherical, then the position angle of the magnetic field in the $\mathrm{S} 1$ shell at the Sun is $P A_{\mathrm{E}} \sim-17^{\circ}$ at the heliosphere nose (Frisch 2008b). This provides evidence that the ISMF in the solar vicinity does not have a purely spherical configuration. ${ }^{1}$

Method (4) above has interesting consequences that are not yet understood. The nature and extent of the TS's distortion depends on both the orientation and strength of the external ISMF. The sources of the heliospheric radio emissions are consistent either with a line roughly parallel to the galactic plane (Kurth and Gurnett 2003) with an average galactic latitude of $14.7^{\circ}$, or with an alternate line parallel to an ecliptic meridian with $\beta \sim 181^{\circ}$ (Frisch 2007). The magnetic field orientation traced by the $3 \mathrm{kHz}$ emissions has been interpreted in different ways. Recently, Gurnett et al. (2006) suggested that the radio emission should occur in regions where magnetic field lines are tangential to the surface of a shock, so that global merged interaction regions instigate particle beams that excite Langmuir waves in the surrounding ISMa plasma (Mitchell et al. 2005; Pogorelov et al. 2008a). If the primary location of the $3 \mathrm{kHz}$ emissions are the correct locations for the origin of these events, this would indicate the ISMF is roughly perpendicular to the galactic plane. A meridinal distribution may instead be due to heliospheric asymmetries.

The direction of the ISMF at the heliosphere has been explored using the faint polarization of nearby stars, which peak in a direction offset along ecliptic longitude by $\Delta \lambda \sim+40^{\circ}$ from the heliosphere nose (Frisch 2007), and the superbubble shell defined by the S1 radio continuum shell (Frisch 2008b; Wolleben 2007). An unconfirmed ENA peak detected in IMAGE data at $\sim 1 \mathrm{keV}$ (Collier et al. 2004; Wurz et al. 2004) is in the same direction of the optical polarization peak. This is shown in Fig. 9. The magnetic field directions traced by the polarizations and the $\mathrm{S} 1$ shell are consistent with the direction of angular offset between

\footnotetext{
${ }^{1}$ The sensitivity of the position angle of the $\mathrm{He}^{\circ}-\mathrm{H}^{\circ}$ offset to the coordinate epoch mistake is illustrated by the value obtained, $P A_{\mathrm{E}}=-32^{\circ}$, by incorrectly comparing $\mathrm{H}^{\circ} \mathrm{J} 2000$ with $\mathrm{He}^{\circ} \mathrm{B} 1950$ coordinates.
} 


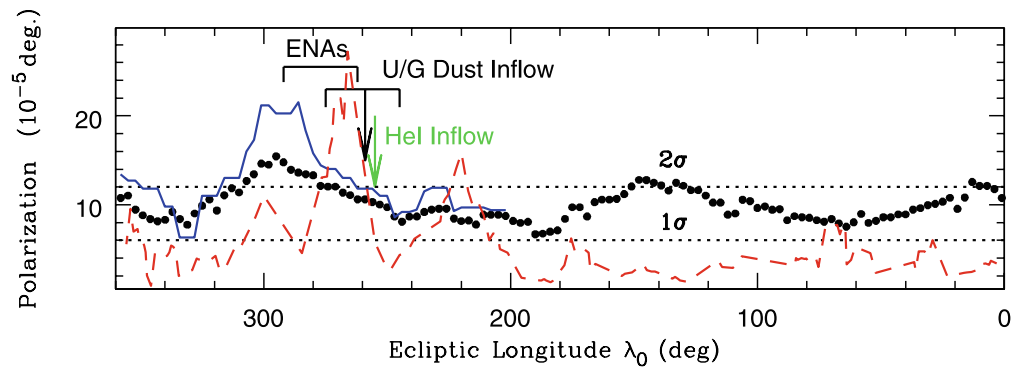

Fig. 9 Polarization properties as a function of ecliptic longitude: The blue line shows the averaged polarizations for stars with $|\beta|<20^{\circ}$, and the dots indicate averaged values for stars with $|\beta|<50^{\circ}$. The $1 \sigma$ and $2 \sigma$ polarization uncertainties are shown (from Tinbergen 1982). The polarization data for nearby stars are averaged over $\pm 20^{\circ}$ in ecliptic longitude, $\lambda$. The direction of peak polarization is shifted by $\sim 40^{\circ}$ from the $\mathrm{He}^{\mathrm{O}}$ upwind direction. The black arrow shows the upstream direction, and $1 \sigma$ uncertainties, for interstellar dust flowing through the heliosphere based on Ulysses and Galileo data (Frisch et al. 1999). The green arrow shows the upstream direction from observations of interstellar $\mathrm{He}^{\mathrm{o}}$ in the inner heliosphere. A tentative upwind direction of an ENA flux from the outer heliosphere is shown (Collier et al. 2004; Wurz et al. 2004), although more recent data do not confirm these earlier conclusions. The red dashed line shows the polarizations, normalized by a factor of 20 and averaged over an interval of $\pm 5^{\circ}$ around the central ecliptic longitude, of 184 stars $40-100 \mathrm{pc}$ from the Sun with $|\beta|<20^{\circ}$

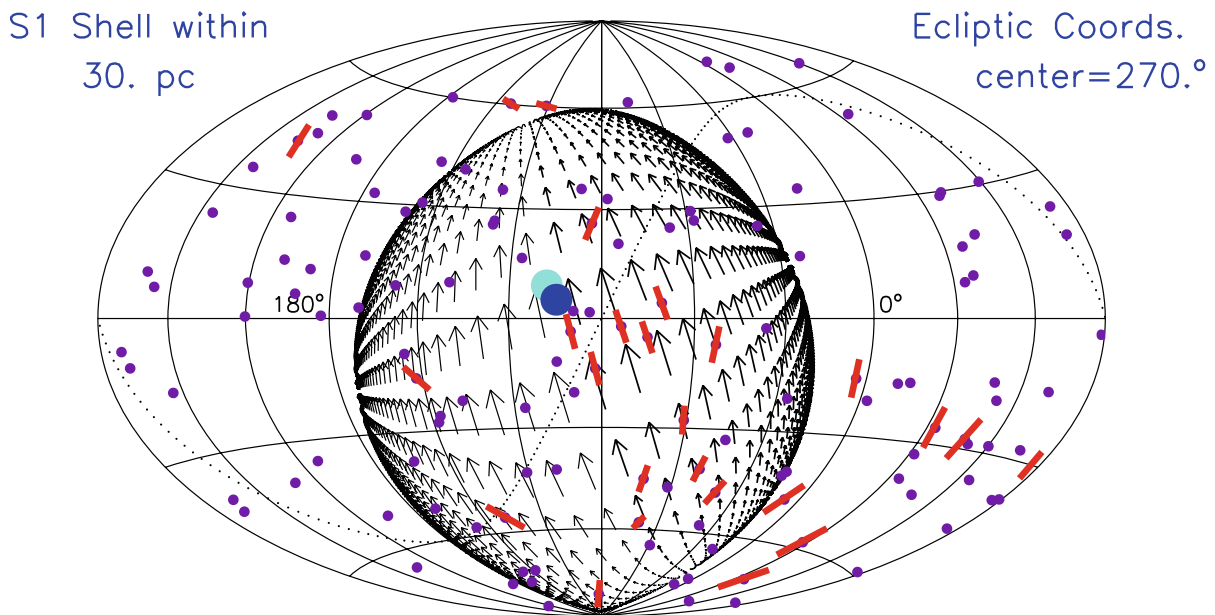

Center $L, B, D=351 .^{\circ},-2 .^{\circ}, 78 . p c \quad$ Radius $=75 . p c \quad$ B_theta,B_phi=71.,$-42 .^{\circ} \quad P>2.5$ sigma

Fig. 10 The magnetic field associated with the parts of the $S 1$ shell (Wolleben 2007) within 30 pc is plotted in ecliptic coordinates for an aitoff projection. The ecliptic plot is centered at $\lambda=270^{\circ}$. The S1 shell parameters have been varied within the range of allowed uncertainties to yield the best match to the polarization data of nearby stars (Tinbergen 1982; Frisch 2007). Dark and light blue dots show the inflow directions of interstellar $\mathrm{H}^{\circ}$ and $\mathrm{He}^{\mathrm{O}}$. The dots show stars within $50 \mathrm{pc}$ with polarization data, and the red bars show polarization vectors for stars where $P>2.5 \sigma$ (Tinbergen 1982; Piirola 1977; Frisch 2007)

the inflowing $\mathrm{H}^{\circ}$ and $\mathrm{He}^{\circ}$ velocities, given the uncertainties (Fig. 10, Frisch 2008b). The upwind direction of the bulk flow of local ISMa past the Sun is within $\sim 10^{\circ}$ of the center of the S1 shell, supporting the conclusion that the S1 shell dominates the ISMa at the Sun.

There are no direct data on the polarity of the local ISMF, so we can only make an educated guess. The Loop I bubble is a distortion of the global local field, which is assumed 
to be the interarm field shown by, e.g., Han (2006). Therefore the near side of this feature, corresponding to the local B-field, is expected to be directed from southern to northern ecliptic latitudes at the solar location if possible magnetic turbulence is ignored.

Frisch $(2007,2008 b)$ found the surprising result that the great circle meridian formed by the plane separating the hot and cold hemispheres of the cosmic microwave background dipole moment, which is generally thought to be dominated by the Doppler motion of the Sun through the cosmic microwave background, is an approximate predictor of the magnetic field direction at the heliosphere nose. This plane is $\sim 5^{\circ}$ from the upwind direction and has a position angle within $\sim 20^{\circ}$ of the magnetic field direction traced by the polarization and $\mathrm{H}^{\circ}-\mathrm{He}^{\mathrm{o}}$ offset data. This combination is unlikely to be random.

\section{The Boundary Conditions of the Heliosphere}

The earliest comparisons between ISMa inside of the heliosphere and towards nearby stars showed that the CHISM is warm, low density, and partially ionized (Rogerson et al. 1973; McClintock et al. 1976; Adams and Frisch 1977). The CHISM properties are regulated by the low average interstellar densities inside of the Local Bubble, and the intensity and spectrum of the interstellar radiation field that follow from our location in a clump of low-opacity ISMa with $N\left(\mathrm{H}^{\mathrm{o}}\right) \lesssim 10^{18.5} \mathrm{~cm}^{-2}$. Frisch and Slavin (2006b) concluded that our location in a tiny clump of ISMa indicates that the heliosphere boundary conditions will vary over timescales of less than $\sim 10^{4} \mathrm{yrs}$, as the Sun crosses the LIC density and ionization gradients at $\sim 26 \mathrm{pc} \mathrm{Myrs}^{-1}$. The boundary conditions of the heliosphere can not be measured directly, but can be modeled by combining ISMa data for a sightline through the LIC with data on interstellar neutrals from inside the heliosphere, combined with estimates of the filtration of interstellar neutrals. Interstellar He has minimal filtration ( $\leq 2 \%$, Sect. 2.1.1) and in situ data on interstellar $\mathrm{He}^{\mathrm{o}}$ provide a valuable constraint on the interstellar densities. These diverse data on local ISMa gain a self-consistent interpretation through the radiative transfer models of the LIC, and together predict the boundary conditions of the heliosphere (Slavin and Frisch 2008).

The heliosphere boundary conditions are sensitive to the spectrum and intensity of the ambient interstellar radiation field, so below we first summarize the significant aspects of this radiation field, including the contamination of the observed soft X-ray background by emission generated by charge exchange between the solar wind and interstellar neutrals. The radiative transfer models of the LIC (Sect. 4.3) and heliospheric $\mathrm{H}^{\circ}$ data (2.1.2), combined with models of the attenuation of interstellar neutrals in the heliosheath regions (or 'filtration'), yield consistent results for the heliosphere boundary conditions.

\subsection{Interstellar Radiation Field and the Heliosphere}

Several spectral intervals of the interstellar radiation field are key to understanding the heliosphere boundary conditions. The first is the $\mathrm{H}$ ionizing radiation field, $E=13.6-24.6 \mathrm{eV}$. The second is the $\mathrm{He}^{\mathrm{o}}$ ionizing field, $E=24.6-54.4 \mathrm{eV}$. Ulysses measurements of $\mathrm{He}^{\mathrm{o}}$ in the heliosphere provide a key constraint on the density of the surrounding ISMa, so that the radiation field capable of ionizing $\mathrm{He}^{\mathrm{o}}$ is important to understanding the heliosphere boundary conditions. Harder radiation, $E>54.4 \mathrm{eV}$ up to $\sim 100 \mathrm{eV}$, including the soft $\mathrm{X}$ ray background radiation field, also may play an important role depending on the strength of the lower-energy ionizing radiation. The soft X-ray background is believed to originate in absorbed distant X-rays (e.g. from the galactic bulge and halo), absorbed X-rays with a 
local origin (e.g. from the Loop I superbubble), and essentially unabsorbed X-ray emission from the $10^{6} \mathrm{~K}$ tenuous, $\sim 0.005 \mathrm{~cm}^{-3}$, LB plasma (e.g. Snowden et al. 2000; Henley et al. 2007), with some foreground contamination from the heliosphere (Sect. 4.2). Models of the total soft X-ray spectrum at the Sun require modeling the temperature and spectrum of all sources.

The far-UV radiation field is also important for understanding the ionization of the LIC, in particular for low first ionization potential elements. Many A, B and late $\mathrm{O}$ stars contribute to the field at the Sun, with the stars in the third and fourth galactic quadrants, $\ell=180^{\circ}-360^{\circ}$ dominating the spatial distribution (Gondhalekar et al. 1980). In contrast the stellar contribution to the extreme ultraviolet (EUV, $E=13.6-100 \mathrm{eV}$ ) radiation field is dominated by the B2 II star $\varepsilon$ CMa (e.g. Vallerga 1996, 1998). New observations by the SPEAR instrument indicate that the diffuse, i.e. dust scattered, portion of the far ultraviolet (FUV) may be considerably larger than previously estimated (Kwang-Il Seon, private communication). If that is the case, the current photoionization models will need to be revised with possible ramifications for the electron density in the LIC and the abundance of C in the LIC.

The lower limit on the EUV radiation field is set by radiation from $\varepsilon \mathrm{CMa}, \beta \mathrm{CMa}$ and nearby white dwarf stars, combined with the low energy tail of the soft X-ray background. The dominant source of EUV emission, however, may originate in the interface between the hot $T \sim 10^{6} \mathrm{~K} \mathrm{LB}$ plasma and the warm LIC. Models of evaporation of the LIC into the LB caused by thermal conduction predict that there is strong EUV emission in a thin layer (Slavin 1989). The detailed results for the dynamical variables such as temperature, density and velocity of the gas as well as the emission in the interface region depend on the orientation and strength of the magnetic field (Slavin 1989; Slavin and Frisch 2008). The interface emission has not yet been directly observed, most of the emission coming in the low end of the EUV where the instruments that have been flown (EUVE, CHIPS) lack sufficient sensitivity for detection. The total radiation field at the Sun, based on a selfconsistent radiative transfer model that includes all of these radiation sources, is shown in Fig. 11.

\subsection{Heliospheric Contamination of the Soft X-Ray Background}

Since the LB affects the LIC via photoionization and direct contact, understanding its spectrum and physical conditions is necessary. In the past, the LB's spectrum was found from shadowing observations, where interstellar clouds located tens to hundreds of pc away block distant X-rays, and the assumption that the LB was the sole source of locally produced diffuse X-rays. Recent observations of solar system X-rays, however, reveal that our solar system is a source of diffuse soft X-rays (Cravens 2000) that contaminate the hot LB's spectrum. These X-rays are emitted from solar wind ions after they have undergone charge exchange with inflowing interstellar neutrals in the solar system. The interaction mechanism is called solar wind charge exchange (SWCX). Here, we provide estimates for the strength and spectrum of the largest SWCX X-ray component, that are due to solar wind ions (excluding those from coronal mass ejections) interacting with neutral interstellar atoms. We also discuss whether SWCX affects our understanding of the photoionization of the LIC. There are two possibilities. First, can these photons directly ionize the CHISM? Second, did ignorance of the SWCX radiation field lead to an overestimation of the flux of the H-ionizing photons originating in the hot LB plasma?

Most of the SWCX emission is formed within 10 AU of the Sun, so that the brightness of heliospheric SWCX X-rays that reach the Earth's location is significant. Robertson and Cravens (2003) estimate the intensity of 0.1 to $1 \mathrm{keV} \mathrm{SWCX} \mathrm{X-rays} \mathrm{found} 1$ AU from the 


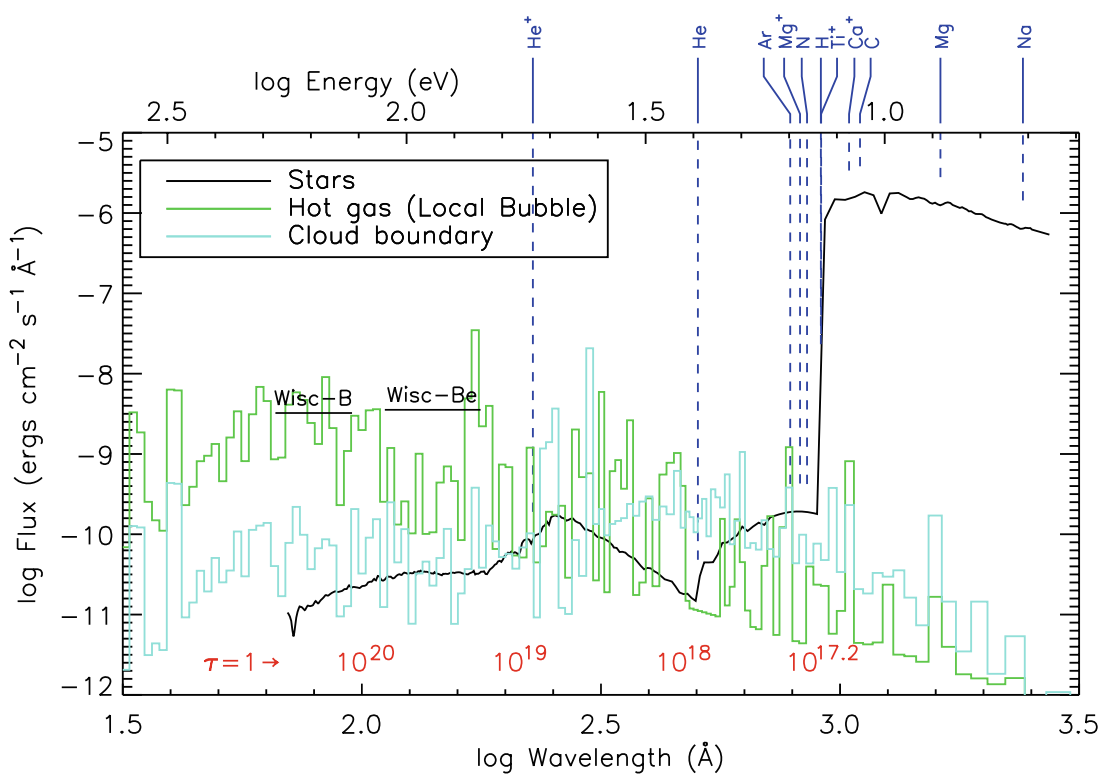

Fig. 11 The interstellar radiation field at the Sun, based on Model 26 in Slavin and Frisch (2008). The lower and top axes show, respectively, wavelength and energy. The green (black) histogram shows the modeled hot gas of the Local Bubble, ignoring possible foreground contamination from charge exchange in the solar wind. Figure 12 suggests that this contamination is negligible. The cyan-blue (gray) histogram is the cloud boundary contribution. The remaining line shows the stellar EUV/FUV background. The list of elements at the top of the plot identifies the ionization potentials for neutrals and ions of interest. The energies/wavelengths at which an optical depth of 1 is reached for several different column densities of $\mathrm{H}^{\circ}$ are shown along the bottom of the plot. Additional information is given in Slavin and Frisch (2008)

Sun to be 3.5 to $6 \mathrm{keV} \mathrm{cm}^{-2} \mathrm{~s}^{-1} \mathrm{sr}^{-1}$, depending on look-direction and phase of the solar cycle. They find that the SWCX X-ray flux accounts for $\gtrsim 1 / 2$ of the X-rays previously attributed to the hot LB plasma. A second set of calculations was performed by Koutroumpa et al. (2008), who concentrated on emission lines in the $3 / 4 \mathrm{keV}$ regime where the LB spectrum is relatively weak, but X-ray emission from SWCX is strong. They found that SWCX may account for all of the O VII and O VIII emission line intensity previously attributed to the hot LB plasma.

At the lower energies important to the LIC ionization, however, SWCX appears to be much less significant. A model spectrum has been provided by Koutroumpa et al. (2008). Fig. 12, top panel, shows the modeled spectrum for an observation of an ecliptic sight line during the maximum phase of the solar cycle. The solar wind is in its slow state in this case. For comparison, Fig. 12, bottom, shows the model spectrum of a thermal gas in collisional ionization equilibrium at a temperature of $10^{6} \mathrm{~K}$, which is the canonical model for the hot LB emission. We have scaled Fig. $12 \mathrm{~b}$ so that it has the same intensity of $\mathrm{O}$ VII triplet $(\sim 570 \mathrm{eV})$ and $\mathrm{O}$ VIII Ly $\alpha(650 \mathrm{eV})$ photons as the SWCX spectrum. While it would be premature to calculate an ionization rate from the SWCX spectrum in Fig. 12, we can note that this SWCX spectrum appears to be harder than the canonical hot LB spectrum, and thus dimmer in the EUV and $\sim 13.6 \mathrm{eV}$ photons most important for ionizing $\mathrm{H}^{\circ}$ in the LIC.

By their nature, SWCX X-ray estimates are uncertain and variable. The chief uncertainties are associated with the abundances of heavy elements in the solar wind, and the cross sections for charge exchange between highly charged ions and neutral material. As a re- 
Fig. 12 Top: Solar wind charge exchange X-ray spectrum, courtesy of Koutroumpa et al. (2008). Bottom: Standard model of the Local Bubble spectrum: that of a thermal plasma in collisional ionization equilibrium at a temperature of $10^{6} \mathrm{~K}$. The lower spectrum has been scaled to yield the same intensity of O VII triplet $(\sim 570 \mathrm{eV})$ and O VIII Ly $\alpha(650 \mathrm{eV})$ photons as the SWCX spectrum

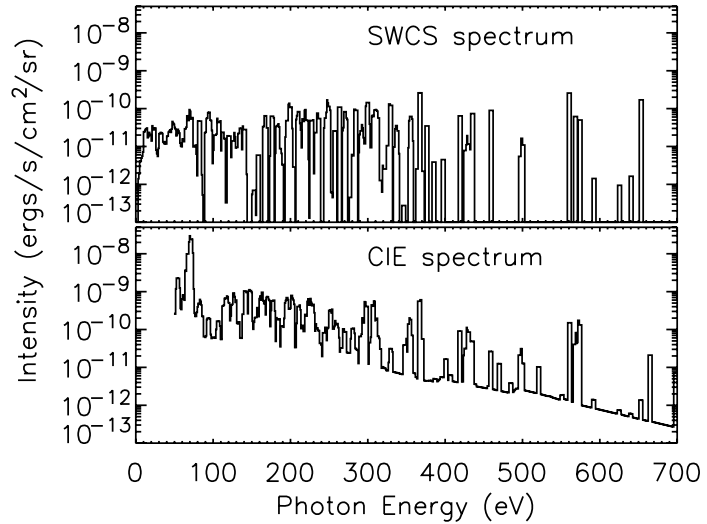

sult, Robertson and Cravens (2003) estimate that their calculation results are uncertain by roughly a factor of 2. In addition, the ionization level, density, and speed of the solar wind vary with the phase of the solar cycle and the location on the Sun (e.g. ecliptic latitude) from which the wind originated. As a result, the SWCX spectrum and intensity vary significantly with look-direction and time.

SWCX photons also are not effective direct ionizers of the LIC for two reasons: First, the SWCX spectrum appears to be harder than that from the hot plasma in the LB, and thus dimmer in EUV photons that could photoionize the LIC. Second, the intensity of SWCX photons affecting the LIC beyond the heliosphere is vastly diluted compared with the intensity of SWCX photons near the Earth and discussed above.

The discovery of SWCX X-rays also affects our understanding of the EUV radiation field capable of ionizing LIC $\mathrm{He}^{\mathrm{o}}$, both through the properties of the conductive interface and the EUV radiation field external to the LIC (Sect. 4.1). If SWCX creates $\sim 1 / 2$ of low galactic latitude $0.25 \mathrm{keV}$ X-ray emission that previously had been attributed to the hot LB plasma, then the LB radiation field may be weaker than previously thought. The theoretical properties of the conductive interface on the LIC have not yet been explored for cases where the LB plasma temperature differs significantly from $10^{6} \mathrm{~K}$, although as of yet there is no evidence for such a difference. One radiation transfer model of the LIC is successful without including interface emission, but most of the models that successfully match the observational constraints on the CHISM do include EUV emission from a conductive interface (Slavin and Frisch 2008). The reduction in the LB's X-ray intensity mandated by the discovery of SWCX X-rays implies that the thermal pressure of the hot gas in the LB is $\sim 1 / \sqrt{2}$ less than previously reported. However, this decrease brings the LB pressure more in line with the LIC pressure used in the radiative transfer models, which assume a LB plasma temperature of $10^{5.9}-10^{6.1} \mathrm{~K}$ and LB thermal pressure of $P / k_{\mathrm{B}} \sim 2000-6600 \mathrm{~cm}^{-3} \mathrm{~K}$.

\subsection{Circumheliospheric Interstellar Material and the Local Interstellar Cloud}

Slavin and Frisch (2008) have evaluated the physical properties of CHISM using a series of radiative transfer models that simultaneously model the properties of the integrated LIC absorption components towards $\varepsilon$ CMa, and the CHISM neutrals traced by in situ heliospheric data. Note that the velocity of the CHISM observed inside of the heliosphere agrees with 
the LIC velocity projected towards $\varepsilon$ CMa. The models are based on a cloud in photoionization equilibrium, using a self-consistent interstellar radiation field which includes, for most models, a conductive interface between the LIC and LB plasma. For those models, the conductive interface is the dominant source of most EUV emission that ionizes $\mathrm{He}^{\mathrm{o}}$ (Sect. 4.1). There presently is no observational proof of the LIC conductive interface, which is predicted to have column densities for O VI on the order of $\log N\left(\mathrm{O}^{+5}\right)=12.3-12.8 \mathrm{~cm}^{-2}$. These values are at the low end of detected O VI column densities towards nearby white dwarf stars that sample the nearest interstellar gas, but there is some debate as to whether the observed features sample interstellar instead of stellar features (Oegerle et al. 2005; Savage and Lehner 2006; Welsh 2008). At least one model is successful without interface emission, and in that case the EUV emission arises in the LB plasma. The LIC opacity is significant near energies of $13.6 \mathrm{eV}$, so the radiation field is propagated self-consistently in the LIC to match radiation field data and interface models. The most useful heliospheric constraints on the CHISM are the velocity, temperature, and $\mathrm{He}^{\mathrm{o}}$ density inside of the heliosphere (Sect. 2.1.1).

The Slavin and Frisch (2008) radiative transfer models yield the following heliosphere boundary conditions: $n\left(\mathrm{H}^{\circ}\right)=0.19-0.20 \mathrm{~cm}^{-3}, n(\mathrm{e})=0.07 \pm 0.01 \mathrm{~cm}^{-3}, T=6340 \mathrm{~K}$, and fractional ionizations for $\mathrm{H}$ and $\mathrm{He}$ of $\chi(\mathrm{H})=\mathrm{H}^{+} /\left(\mathrm{H}^{\circ}+\mathrm{H}^{+}\right)=0.19-0.26$ and $\chi(\mathrm{He})=$ $\mathrm{He}^{+} /\left(\mathrm{He}^{\circ}+\mathrm{He}^{+}\right)=0.36-0.43$. These results are in excellent agreement with independent estimates of $n\left(\mathrm{H}^{\circ}\right)$ and $n\left(\mathrm{H}^{+}\right)$based on observations of $\mathrm{H}$ pickup ions, combined with heliosphere models that predict $\mathrm{H}$ filtration (Sect. 2.1.2, Bzowski et al. 2008). The interstellar electron density in the CHISM is expected to be somewhat lower than the sightline averaged value, because of radiative transfer effects, and higher values are found towards stars sampling other parts of the CHISM (Sect. 5.3.3).

The key constraints on the total LIC electron density are the interstellar ratios of $\mathrm{Mg}^{\circ} / \mathrm{Mg}^{+}$and $\mathrm{C}^{+*} / \mathrm{C}^{+}$. The key constraints on the CHISM neutrality, and therefore ionization, are data on interstellar neutrals in the heliosphere, particularly $\mathrm{He}^{\circ}$, and also $\mathrm{H}, \mathrm{O}$, $\mathrm{N}, \mathrm{Ne}$, and Ar pickup ions. Better values for the filtration of $\mathrm{O}, \mathrm{N}, \mathrm{Ne}$, and Ar are desirable to improve the comparisons between models and in situ data. The data constraining the models, and the model predictions, are listed in Table 1 . The success of the radiative transfer models in reproducing LIC data supports the assumption that the surrounding cloud is in photoionization and thermal equilibrium. The modeling procedure is sensitive to the $\mathrm{He}^{\mathrm{o}}$ density inside the heliosphere.

The radiative transfer models also provide deeper insight into the heating and cooling mechanisms, as well as the chemical composition and dust mass, of the LIC. Heating of the LIC is dominated by the photoionization of $\mathrm{H}^{\circ}(\sim 66 \%)$ and $\mathrm{He}^{\circ}(\sim 25 \%)$. Cooling is dominated by the emission from the collisionally excited fine-structure lines of $\mathrm{C}^{+}(\sim 43 \%)$ and other ions $(\sim 35 \%)$. In the LIC, carbon appears to be overabundant by a factor of $2-3$, possibly indicating destruction of carbonaceous grains in interstellar shocks (Slavin and Frisch 2006, 2008). The LIC abundances of refractory elements found from these radiative transfer models are typical abundances for interstellar clouds where dust grains have been destroyed by processing through interstellar shock fronts, i.e. the Routly-Spitzer effect (Routly and Spitzer 1952; Jones et al. 1994), and in the case of the LIC indicate grain processing by a shock with velocity $\sim 90-140 \mathrm{~km} \mathrm{~s}^{-1}$ (Frisch et al. 1999). A problem with this interpretation is that models (e.g., Jones et al. 1994) predict that silicate dust would be more destroyed than carbonaceous dust. Dust destruction in shocks remains an active area of research that these results on the LIC can help to guide.

These models can also be used to predict the gas-to-dust mass ratio at the heliosphere boundary, and those predictions can be compared with values determined from in situ observations of dust in the heliosphere (Sect. 2.3). The prediction of $R_{\mathrm{g} / \mathrm{d}}$ from the radiative 
Table 1 Neutral densities at heliosphere boundary from in situ data and models

\begin{tabular}{llll}
\hline Element & $\begin{array}{l}\mathrm{TS}^{*} \\
\mathrm{~cm}^{-3}\end{array}$ & $\begin{array}{l}\text { Interstellar } \\
\mathrm{cm}^{\dagger}\end{array}$ & $\begin{array}{l}\text { Filtration }^{\dagger} \\
\text { Ratios }\end{array}$ \\
\hline $\mathrm{H}^{\circ}$ & $0.11 \pm 0.02$ & $0.194 \pm 0.008$ & $\sim 0.56$ \\
$\mathrm{He}^{\mathrm{o}}$ & $0.015 \pm 0.003$ & $0.015 \pm 0.001$ & {$[1]^{\diamond}$} \\
$\mathrm{N}^{\circ}$ & $5.47 \pm 1.37 \times 10^{-6}$ & $8.90_{-1.99}^{+2.80} \times 10^{-6}$ & $0.68-0.95$ \\
$\mathrm{O}^{\circ}$ & $4.82 \pm 0.53 \times 10^{-5}$ & $7.16_{-1.45}^{+2.14} \times 10^{-5}$ & $0.64-0.99$ \\
$\mathrm{Ne}^{\circ}$ & $5.82 \pm 1.16 \times 10^{-6}$ & $5.20_{-0.67}^{+0.79} \times 10^{-6}$ & $0.84-0.95$ \\
$\mathrm{Ar}^{\circ}$ & $1.63 \pm 0.73 \times 10^{-7}$ & $1.88_{-0.25}^{+0.16} \times 10^{-7}$ & $0.53-0.95$ \\
\hline
\end{tabular}

* Densities at the termination shock are based on pickup ion and $\mathrm{He}^{\mathrm{O}}$ data (Gloeckler and Fisk 2007; Bzowski et al. 2008; Möbius et al. 2004)

$\dagger$ Interstellar densities are based on radiative transfer models 14, 26-42, in Table 4 of Slavin and Frisch (2008)

\$ The filtration ratio is the percentage of the neutral that survives traversal of the heliosheath regions

The source for these filtration ratios is listed in Slavin and Frisch (2008)

$\diamond \mathrm{He}^{\mathrm{O}}$ was assumed to have no filtration (although up to $\sim 2 \%$ filtration is possible, Müller et al. 2004)

transfer models requires some assumption about the underlying abundances in the CHISM. The ACR isotopic data (Sect. 3.1) suggest that the surrounding interstellar cloud has solar composition. If solar abundances from Lodders (2003) are used, then we find $R_{\mathrm{g} / \mathrm{d}} \sim$ 160-240 for the CHISM, versus values $R_{\mathrm{g} / \mathrm{d}}<115-125$ that are determined using in situ observations of the interstellar dust inside of the heliosphere (Slavin and Frisch 2008). The origin of the difference between these two values is not yet understood, but may indicate decoupling of interstellar gas and dust over very small spatial scales.

\section{Complex of Local Interstellar Clouds}

The Sun is embedded in a flow of ISMa that moves through the local standard of rest (LSR) with a bulk motion of $\sim 17 \mathrm{~km} \mathrm{~s}^{-1}$ (Frisch and Slavin 2006a). The kinematics of this flow are determined by diffuse clouds in front of stars within $30 \mathrm{pc}$, and is typical of low-density interstellar clouds. In contrast, low velocities of $\sim 0-2 \mathrm{~km} \mathrm{~s}^{-1}$ are found for more distant, cold, dense molecular clouds. Fast, low-density ISMa has long been known to show enhanced abundances of $\mathrm{Ca}^{+}$and other refractory elements, caused by the destruction of dust grains in interstellar shocks (Routly and Spitzer 1952; Jones et al. 1994). Based on the kinematics and enhanced refractory abundances measured for ISMa within $14 \mathrm{pc}$, Frisch (1981) originally proposed that the CLIC is part of the Loop I superbubble that has expanded to the solar location. More recently Wolleben (2007) used $1.4 \mathrm{GHz}$, radio-continuum-polarization data and found that the Sun is located in the rim of the S1 subshell of Loop I (see Figs. 10 and 13).

\subsection{Dynamics of the Complex of Local Interstellar Clouds}

Two approaches have been used to study the dynamics of local ISMa: (1) The bulk motions of the ensemble of nearby clouds have been derived from some combination of optical and UV data (Crutcher 1982; Frisch and York 1986; Bzowski 1988; Vallerga et al. 1993; Frisch 
et al. 2002). (2) Individual dynamical clumps in the flow of ISMa past the Sun have been identified based on pure kinematics (Frisch 1981; Lallement et al. 1986; Frisch et al. 2002), or on both kinematical and temperature properties (Redfield and Linsky 2008). Many papers report cloud velocities in the solar system barycenter. However, for proper comparison with structural features in the global ISMa, velocities must be converted into the LSR.

Ultimately, all identifications of nearby interstellar clouds rely on absorption lines interpreted with the assumption that the absorbing gas has a thermal distribution of velocities around a central velocity, combined with non-thermal microturbulence. Absorption line spectroscopy is a powerful technique that, due to the Doppler shift of interstellar structures, can measure several distinct absorption components along a single line of sight. This technique has been used extensively, particularly at high spectral resolution (e.g. Crawford 2001; Welty et al. 1999; Gry and Jenkins 2001; Crawford 2001; Redfield and Linsky 2002, 2004a; Welty et al. 2003; Frisch et al. 2002). One basic property of LISM dynamics is that most sightlines show multiple distinct absorption profiles, particularly near the upwind-downwind axis where the radial components of the Doppler motions dominate. This argues for structured, coherently moving collections of gas in the LISM. For example, the median difference in radial velocity for LISM clouds within $100 \mathrm{pc}$ and along a single line of sight is $\sim 7.5 \mathrm{~km} \mathrm{~s}^{-1}$, whereas the typical line width (including thermal and microturbulent broadening mechanisms) is less than half of that at $\sim 2.8 \mathrm{~km} \mathrm{~s}^{-1}$ (Redfield and Linsky 2004a). One fact not yet understood about interstellar absorption lines is that the number of identified components increases as the spectral resolution of the observations increases. Welty and collaborators (Welty et al. 1996a; Welty and Hobbs 2001) argue that $\sim 40-48 \%$ of absorption components in distant sightlines, where the average number of absorbers is 5.0-9.5 per sight line, remain undetected because they are severely blended and observed at too low of a spectral resolution. For the LISM, on average, there are only 1.7 absorbers per sight line (Redfield and Linsky 2004a), yet absorption components are generally blended in velocity.

The recent studies of the kinematical structure of the LISM, described below, present the same general picture but do not agree in the detailed descriptions of cloudlets. Future studies of this topic need to include high-resolution observations in the UV, that can simultaneously provide information on the ionization, neutral densities, and kinematical structure of each sightline.

\subsubsection{Bulk Dynamics of Local Interstellar Gas}

Various studies of the CLIC properties and dynamics have concluded that the LISM within $\sim 30$ pc flows away from the central regions of the Loop I supernova remnant (Frisch 1981; Crutcher 1982; Frisch and York 1986; Bzowski 1988; Vallerga et al. 1993; Frisch et al. 2002). Recent studies sampling ISMa within $\sim 30 \mathrm{pc}$ show that the bulk flow of ISMa past the Sun has an LSR vector motion of $-17 \mathrm{~km} \mathrm{~s}^{-1}$ from the upwind direction of $(\ell, b) \sim$ $\left(358^{\circ},-5^{\circ}\right)$. This LSR upwind direction of the CLIC is near the center of the S1 subshell of the Loop I supernova remnant, at $\ell=346^{\circ} \pm 5^{\circ}, b=3^{\circ} \pm 5^{\circ}$, identified by Wolleben (2007) based on $1.4 \mathrm{GHz}$ polarization data. This LSR upwind direction, combined with the solar location inside the shell rim, suggest the bulk flow velocity vector represents the mean motion of the S1 shell expanding past the Sun. Fig. 13 shows the bulk motion of the CLIC through the LSR, together with the solar apex motion and the S1 shell location. The S1 center distance $(78 \pm 10 \mathrm{pc})$ and rim thickness $(\sim 20 \mathrm{pc})$ place the Sun inside of the shell rim. Comparisons of CLIC velocities for stars within $\sim 10 \mathrm{pc}$ show that the bulk flow of ISMa past close to the Sun is decelerating (Frisch and York 1986; Redfield and Linsky 


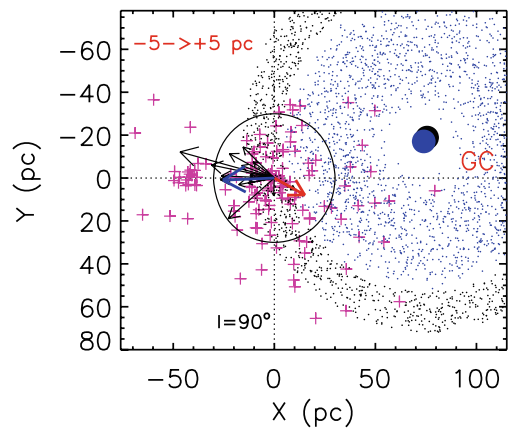

Fig. 13 Slices of the $\mathrm{S} 1$ shell (black dots, large ring) and $\mathrm{S} 2$ shell (blue dots, small ring) within $5 \mathrm{pc}$ of the galactic plane $(|Z|<5 \mathrm{pc})$. The shell properties are displayed in Cartesian coordinates with the galactic center at the right and the direction of LSR rotation at the bottom. See Wolleben (2007) and Frisch (2008b) for additional information on the S1 and S2 shells. The red and blue arrows show the LSR velocities of the Sun and CLIC from Frisch and Slavin (2006a), and the black arrows show the 15 cloudlets of Redfield and Linsky (2008, RL8). The black circle shows the $30 \mathrm{pc}$ radius region represented by the CLIC bulk flow vector. The pink crosses show the locations of the stars used to derive the velocity model with 15 cloudlets. The dot density scales with column density through the S1 and S2 slices. The coldest portions of the S1 shell are also seen as arcs protruding from the Scorpius-Centaurus Association in Fig. 2. This cold gas is observed, for instance, in $\mathrm{Na}^{\circ}$ absorption towards $\delta$ Cyg (e.g. Hobbs 1969)

2001; Frisch et al. 2002). This effect vanishes when more distant stars, within $\sim 100$ pc, are included.

\subsubsection{Structure and Cloudlets in the Complex of Local Interstellar Clouds}

The inhomogeneous LISM was first apparent in individual 'clouds' identified 45 years ago towards stars within 20 pc (Munch and Unsold 1962; Hobbs 1969). The first spectrum of interstellar gas inside of the heliosphere was a Copernicus observation of the $\mathrm{H}^{\circ}$ Ly $\alpha$ backscattered emission obtained during 1975, which determined an $\mathrm{H}^{\circ}$ velocity of $\sim-24.7 \mathrm{~km} \mathrm{~s}^{-1}$ (neglecting heliospheric acceleration and after correcting for the recent Ulysses measurement of the $\mathrm{He}^{\mathrm{o}}$ upstream direction, Sect. 2.1.1, Adams and Frisch 1977; Frisch 2008a). The nearest star for which optical data were then available, $\alpha$ Oph at $14 \mathrm{pc}$, showed interstellar $\mathrm{Ca}^{+}$velocities of $-24 \mathrm{~km} \mathrm{~s}^{-1}$ and $-26 \mathrm{~km} \mathrm{~s}^{-1}$ (Marschall and Hobbs 1972; Crawford 2001). In contrast, the heliospheric $\mathrm{H}^{\circ}$ velocity projected in the $\alpha \mathrm{Oph}$ direction was $\sim-21.1 \mathrm{~km} \mathrm{~s}^{-1}$. Although some of that difference is now recognized to be due to the deceleration of $\mathrm{H}^{\circ}$ at the hydrogen wall (Sect. 3.2), this mismatch became the first evidence of velocity inhomogeneities in the LISM.

The earliest studies of the LISM structure were based on the kinematics of absorption components identified towards individual nearby stars. Frisch (1994) assumed that the LIC velocity vector is parallel to a cloud surface normal and used ISMa data towards $\alpha \mathrm{CMa}$ at $2.7 \mathrm{pc}$ to estimate that the Sun has entered the LIC within the past several thousand years. Using the original definition of the LIC velocity, Lallement et al. (1995) compared the ISMa velocity inside the heliosphere with ISMa velocities towards upwind stars, including $\alpha$ Cen at $1.3 \mathrm{pc}$, and concluded that the Sun will emerge from the LIC in the next 10,000 years. Again using the original definition of the LIC velocity, the absence of a LIC component towards the upwind stars (Wood et al. 2000b) and $\alpha$ Cen (the nearest star, Linsky and Wood 1996) set an upper limit for the distance to the LIC edge in the upwind direction of $\sim 0.1 \mathrm{pc}$. These studies then suggested that the Sun is inside of a tiny distinct kinematic structure, and will 
exit this kinematical structure in $\leq 3,800 \mathrm{yrs}$. The most recent definition of the LIC velocity by Redfield and Linsky (2008, see below), using absorption components towards stars up to $100 \mathrm{pc}$ away, instead places the Sun between two cloudlets. In this later case, the CHISM itself would be an independent kinematic structure, sampled only by the heliosphere. At this point the discussion enters the philosophical territory as to "what is an interstellar cloud" (a question posed by Eugene Parker). We note that 30 years ago 'clouds' such as the LIC and CHISM were referred to as 'intercloud material'.

A kinematical study of the whole sky requires both high-resolution optical $\mathrm{Ca}^{+}$data and medium-resolution UV absorption lines, because the UV resonance lines are far more opaque than $\mathrm{Ca}^{+}$and thus allow measurements of warm ISMa in the galactic anticenter and north galactic pole directions where $\mathrm{Ca}^{+}$column densities are very low. Individual cloudlets have been identified in the bulk flow of ISMa past the Sun based on the fitting of clouds with thermal Maxwellian velocity distributions combined with non-thermal microturbulence. Based on kinematics, Lallement et al. (1986) identified five cloudlets in the CLIC. The subsequent inclusion of UV absorption lines showed that the gas flow in the galactic anticenter hemisphere can be fit with a single velocity vector (originally called the AG Cloud and now called the LIC flow vector), but the galactic center hemisphere required a slightly different velocity vector (called the G Cloud flow vector). A more extended set of data, focused on stars that traced ISMa within $30 \mathrm{pc}$, allowed identification of seven distinct kinematical structures (Frisch et al. 2002). One difficulty in assigning absorption components to cloudlets in the CLIC is that many features have similar upwind directions, e.g. the LIC and G-cloud, yielding ambiguous assignments for stars located $\sim 90^{\circ}$ from the bulk flow direction.

A more detailed cloudlet structure based on kinematical arguments has been developed by Redfield and Linsky (2008), who assembled a database with 270 radial-velocity measurements for 157 sightlines towards stars located within $100 \mathrm{pc}$, including the data from Lallement et al. (1986) and Frisch et al. (2002). The locations of these stars are plotted in Fig. 13, and include sightlines that sample both the S1 and S2 shells of Wolleben (2007). About $55 \%$ of this database consists of velocities measured in UV absorption lines of $\mathrm{D}^{\circ}$, $\mathrm{C}^{+}, \mathrm{O}^{\circ}, \mathrm{Si}^{+}, \mathrm{Mg}^{+}, \mathrm{Fe}^{+}$, measured by the GHRS and STIS instruments on HST, and $45 \%$ of the data are from ground-based $\mathrm{Ca}^{+}$spectra. Nearly half of these radial velocities, primarily in the galactic anticenter hemisphere, are consistent with a single flow vector to within $\pm 1 \mathrm{~km} \mathrm{~s}^{-1}$, which has also been named the LIC, and is denoted here the $V_{\mathrm{LIC}, \mathrm{RL}}$. The $V_{\mathrm{LIC}, \mathrm{RL}}$ flow vector in the LSR has a velocity amplitude of $23.8 \pm 0.9 \mathrm{~km} \mathrm{~s}^{-1}$, and is directed towards galactic coordinates $l=187.0^{\circ} \pm 3.4^{\circ}$ and $b=-13.5^{\circ} \pm 3.3^{\circ}$. Alternate definitions of the LIC velocity vector are based on the velocity of interstellar $\mathrm{He}^{\mathrm{o}}$ inside of the heliosphere (Sect. 2.1.1) and interstellar absorption components in nearby stars in the downwind direction (Lallement and Bertin 1992). The temperatures of the components assigned to the $V_{\text {LIC,RL }}$ component vary from $5,200 \mathrm{~K}\left(\kappa^{1} \mathrm{Cet}\right)$ to $12,050 \mathrm{~K}$ (V368 Cep), with a weighted mean of $7500 \pm 1300 \mathrm{~K}$, based on 19 independent temperature measurements. Temperatures are determined by assuming mass-dependent thermal and mass-independent turbulent broadening of absorption lines, with the low mass measurements generally based on the $\mathrm{D}^{\circ}$ Ly $\alpha$ absorption line, which is superimposed on the blue wing of the stronger $\mathrm{H}^{\circ} \mathrm{Ly} \alpha$ feature.

The morphology of the LIC, according to Redfield and Linsky (2008), is shown in Fig. 14. Using components with radial velocities that are inconsistent with the $V_{\text {LIC,RL vec- }}$ tor, Redfield and Linsky (2008) then searched for patches of galactic coordinates that contain radial velocities consistent with different velocity vectors to within measurement errors. The largest such patch is the $\mathrm{G}$ cloud based on 21 sightlines, but there are 13 other clouds each based on $4-15$ sightlines. All of these clouds lie within $15 \mathrm{pc}$ of the Sun as determined by 


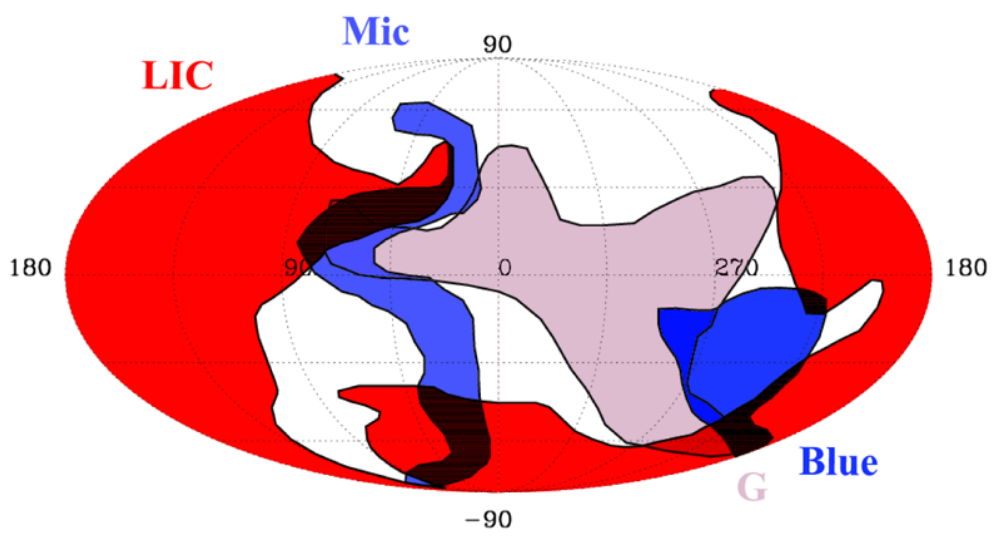

Fig. 14 The location in galactic coordinates of four interstellar clouds that are closest to the heliosphere. The Mic Cloud, which is likely located between the LIC and G Clouds with a similar shape, may be compressed and heated by the collision of the LIC and G Clouds. The G Cloud is seen in front of $\alpha$ Cen (1.3 pc), and the Blue Cloud is seen in front of Sirius $(2.6 \mathrm{pc})$. Eleven other warm clouds located within $15 \mathrm{pc}$ of the Sun are not plotted, but together the 15 clouds cover the whole sky (Redfield and Linsky 2008)

the nearest star with an interstellar velocity component consistent with the cloud vector. By this criterion, six of the clouds are closer than $3.5 \mathrm{pc}$, and the $\mathrm{G}$ cloud lies within $1.3 \mathrm{pc}$, the distance to $\alpha$ Cen. Approximately $19 \%$ of the velocity components could not be assigned to a cloud, indicating that they are located in a poorly sampled region of the sky or that the absorbing gas cloud subtends too small an angle to determine a reliable velocity vector either because the cloud is intrinsically small or too far away. Half of these 15 cloudlets overlap kinematical entities identified in earlier studies (Frisch 1981; Lallement et al. 1986; Frisch et al. 2002), and the differences between the original and newly derived vectors show that the three-dimensional kinematics of a cloud are highly sensitive to the set of stars, and absorption components, that have been selected to identify the clouds.

The velocity of ISMa inside of the heliosphere is well established by interstellar $\mathrm{He}^{\mathrm{o}}$ flowing into the heliosphere at $-26.3 \pm 0.5 \mathrm{~km} \mathrm{~s}^{-1}$ (Sect. 2.1.1), which is between the flow speed of the $V_{\text {LIC,RL }}\left(-23.8 \pm 0.9 \mathrm{~km} \mathrm{~s}^{-1}\right)$ and the G Cloud $\left(-29.6 \pm 1.1 \mathrm{~km} \mathrm{~s}^{-1}\right)$. When the new velocity vector $V_{\mathrm{LIC}, \mathrm{RL}}$ is used, the direction of the inflowing $\mathrm{He}^{\mathrm{o}}$ is intermediate between that of the G and RL8-LIC clouds, and the temperature of the inflowing $\mathrm{He}^{\mathrm{o}}$ gas $(6300 \pm 390 \mathrm{~K}$, Witte 2004; Möbius et al. 2004) is also intermediate between the RL8-LIC $(7500 \pm 1300 \mathrm{~K})$ and the G Cloud $(5500 \pm 400 \mathrm{~K})$. Redfield and Linsky (2008) therefore concluded that heliosphere is in the transition region between the LIC and G Clouds. In this case, however, the CHISM is seen nowhere except inside of the heliosphere.

The identification of distinct, rigidly moving, kinematically defined cloudlets in the CLIC flow is subject to several different uncertainties. As noted above, the similar upwind directions for most of the local cloudlets identified so far means that absorption components towards stars in the sidewind direction frequently have velocities consistent with more than one cloudlet. A second uncertainty follows from apparent deceleration of the nearest parts of the CLIC. In addition, many cloud identifications rest on blended components where uncertainties in component velocities are comparable to the velocity differences between the cloudlets. An example of this later case is the sightline towards $\alpha$ Oph. High-resolution observations of the most positive absorption component towards $\alpha \mathrm{Oph}$, at $14 \mathrm{pc}$, find heliocentric velocities that differ by several $\mathrm{km} \mathrm{s}^{-1}$ for components forming $V_{\mathrm{LIC}, \mathrm{RL}}$, with values of $-22.2 \mathrm{~km} \mathrm{~s}^{-1}$ (Welty et al. 1996b), $-23.6 \mathrm{~km} \mathrm{~s}^{-1}$ (Crawford and Dunkin 1995), and 
$-24.0 \mathrm{~km} \mathrm{~s}^{-1}$ (Crawford 2001). The uncertainty range of this component velocity is comparable to the difference of $\sim 2.4 \mathrm{~km} \mathrm{~s}^{-1}$ between the interstellar $\mathrm{He}^{\mathrm{o}}$ velocity and $V_{\mathrm{LIC}, \mathrm{RL}}$, projected in the $\alpha$ Oph direction. These various uncertainties can only be overcome by building a comprehensive high-resolution database of ISMa within $30 \mathrm{pc}$, inclusive of UV spectral data, so that cloud physical properties can also be investigated.

\subsection{Origin of Local Interstellar Medium}

The similarity in the upwind directions of the velocity vectors of LISM clouds argues for a collective history or driver in order to create the common motion of LISM clouds. Using information on the dynamics and abundances of nearby warm gas, Frisch (1981) originally found that the origin of local ISMa is directly related to the history and structure of the asymmetrically expanding Loop I bubble formed by the supernovae and winds from stars in the Scorpius-Centaurus Association. The averaged LSR upwind direction of LISM cloudlets is found to be $l \sim 357.8^{\circ}, b \sim-5.2^{\circ}$ using stars within $30 \mathrm{pc}$ (Frisch and Slavin 2006a) or $l \sim 10.7^{\circ}, b \sim 16.6^{\circ}$ using stars within $100 \mathrm{pc}$ (Redfield and Linsky 2008). These directions are coincident with the location of one of the nearest OB associations, the ScorpiusCentaurus Association, and within 20 degrees of the Upper Scorpius subgroup. The agreement is even stronger for the location of the associations when they were formed, from 5-10 Myr ago (de Geus et al. 1989; Maíz-Apellániz 2001), when the upwind direction is within 10 degrees of both the Upper Scorpius and Upper Centaurus Lupus subgroups. A related interpretation of the data is that the LISM is part of the S1 shell (Fig. 13, and Frisch 2008b).

The connection between Sco-Cen and the Local Bubble, as well as the warm LISM around the Sun, has been discussed by various authors (e.g. Frisch 1981, 1995; Frisch and York 1986; Bochkarev 1987; Maíz-Apellániz 2001; Fuchs et al. 2006; Wolleben 2007; Redfield and Linsky 2008). Shells swept up by the winds of young stars in the ScorpiusCentaurus Association may be coincident with the location of the Sun today, and may be responsible for the very local warm ISMa clouds observed in our immediate vicinity. Frisch (1995, 1996) modeled the Loop I shell at the Sun as formed 4-5 Myrs ago by stellar evolution in the Scorpius-Centaurus Association. Breitschwerdt and de Avillez (2006) proposed an alternative, but related, model where supernovae from a Pleiades subgroup led to the formation of the LB. The interaction of two such structures has led to theories of the formation of warm LISM clouds from Rayleigh-Taylor instabilities near the interaction point, which subsequently get driven by the prevailing flows from the Scorpius-Centaurus Association (Breitschwerdt et al. 2000; Egger and Aschenbach 1995). Finally, straightening magnetic flux tubes, pulling dense material from the LB boundary, has been discussed as a possible formation mechanism for the warm LISM clouds by Cox and Helenius (2003). Detailed morphological and physical models of both the warm LISM clouds and the hot gas in the LB need to be assembled in order to develop a coherent model for the origin and evolution of our most local interstellar environments.

\subsection{Physical Properties of the Local Interstellar Medium}

\subsubsection{Temperature and Turbulence}

High-resolution UV spectra obtained with the GHRS and STIS instruments on HST have provided the important data for inferring the physical properties of the clouds. The widths of interstellar absorption lines of ions with atomic masses differing by over an order of magnitude, e.g. $\mathrm{D}^{\circ}$ and $\mathrm{Fe}^{+}$, can in principle be used to separate purely thermal from nonthermal (turbulent) broadening components. This deconvolution of line widths is based 
on the assumption that the line full-width-half at half-maximum is given by FWHM $1.7 * \sqrt{V_{\text {thermal }}^{2}+V_{\text {turbulent }}^{2}}$, where $V_{\text {thermal }}$ represents the mass-dependent Doppler broadening of the line by thermal motions, and $V_{\text {turbulent }}$ is presumably mass-independent line broadening due to the turbulent motions. We note that this later assumption may break down for ions subject to magnetically-induced gyromotion. Redfield and Linsky (2004b) found a weighted mean temperature along fifty LISM sightlines of $6680 \pm 1490 \mathrm{~K}$, with a range between $1000 \mathrm{~K}$ and $12,500 \mathrm{~K}$. The weighted mean nonthermal broadening is $2.24 \pm 1.03 \mathrm{~km} \mathrm{~s}^{-1}$, which is subsonic and provides less pressure support than the thermal component. The mean turbulence may be somewhat overestimated due to unresolved velocity components. Redfield and Linsky (2004b) also found a moderately significant negative correlation of $T$ with nonthermal broadening, which, if real, could result from pressure equilibrium. A puzzling aspect of the negative correlation is found for stars within $10 \mathrm{pc}$, where the negative correlation between temperature and turbulence is accompanied by positive correlations between $N\left(\mathrm{D}^{\circ}\right)$ and temperature (Frisch 2008a). In low-density, partially ionized ISMa such as the LIC, $\mathrm{D}^{\circ}$ and $\mathrm{Fe}^{+}$will have different spatial distributions, so that radiative transfer models of the sightline are required to accurately compare abundances of these species with different ionization levels.

\subsubsection{Chemical Composition}

In principle, elemental abundances can be used to estimate depletions onto dust grains, based on some assumed reference abundance for the cloud. Isotopic abundances suggest that solar abundances are appropriate for the CHISM (Sect. 2.2). Abundances in partially ionized gas must be compared to $N\left(\mathrm{H}^{\circ}+\mathrm{H}^{+}\right)$, as has been done implicitly for the CHISM through the use of radiative transfer models (Sect. 4.3). However cloud ionizations are not known for most of the CLIC sightlines. In the CHISM, gas-phase, refractory-element abundances of $\mathrm{Fe}, \mathrm{Mg}$, and $\mathrm{Si}$ are enhanced over cold cloud values by factors of $\sim 12, \sim 3$, and $\sim 4$, which is attributed to the destruction of ISDGs in shocks (Jones et al. 1994; Frisch et al. 1999; Slavin and Frisch 2008). Redfield and Linsky (2008) found that small depletions of Fe and $\mathrm{Mg}$ are correlated with high turbulence values. A further consideration is that since the Sun resides in the rim of the S1 shell, and the CLIC samples the S1 shell (Fig. 13), it is likely that depletions are similar throughout the shell. In this case, apparent local depletion variations would either be due to neglected ionization corrections, or the above-mentioned problems with the conventional line-broadening algorithm.

\subsubsection{Ionization}

Ionization has been determined for the LISM from collisionally excited $\mathrm{C}^{+*}$ lines and the $\mathrm{Mg}^{\circ}$ line, which has a strength enhanced in warm gas $(>6000 \mathrm{~K})$ by dielectric recombination (York and Kinahan 1979). The range of interstellar electron densities found for gas within $\sim 100 \mathrm{pc}$, using either the $\mathrm{Mg}^{\circ} / \mathrm{Mg}^{+}$or $\mathrm{C}^{+*} / \mathrm{C}^{+}$ratios, is $n(\mathrm{e})=0.02-0.50 \mathrm{~cm}^{-3}$ (Frisch et al. 1990; Lallement and Ferlet 1997; Lehner et al. 2003; Redfield and Falcon 2008). The CHISM value $n(\mathrm{e})=0.07 \pm 0.01 \mathrm{~cm}^{-3}$ determined from radiative transfer models (Sect. 4.3) is thus consistent with the general values in the LISM. The nearest star with a high observed level of ionization is the white dwarf HD 149499B (37 pc), where $N\left(\mathrm{~N}^{+}\right) / N\left(\mathrm{~N}^{\circ}\right) \sim 2$ (Lehner et al. 2003). The ionizations levels of $\mathrm{N}$ and $\mathrm{H}$ are coupled by charge exchange in diffuse clouds. 


\subsubsection{When Local Clouds Interact}

We now know that even over sightlines as short as a few parsecs there are on average 1.7 clouds. Redfield and Linsky (2008, RL08) estimated that warm clouds occupy 5.5-19\% of the volume of space within $15 \mathrm{pc}$. The model with 15 LISM velocity vectors (Sect. 5.1.2) allowed RL08 to compute the relative velocity differences between clouds located along the same sightline. These velocity differences are often supersonic $\left(>8 \mathrm{~km} \mathrm{~s}^{-1}\right)$ and in some cases as large as $45 \mathrm{~km} \mathrm{~s}^{-1}$, although we do not know the locations of most clouds along lines of sight. These pieces of information make it likely that some clouds interact with each other and that the collisions can produce shocks. Linsky et al. (2008) showed that the three quasars with well-studied, large-amplitude intraday and annual scintillation variability are all located along lines of sight that pass through the edges of warm clouds where cloud-cloud interactions are likely. Since scintillation is produced by a turbulent ionized medium, this study reinforces the possibility that locally cloud-cloud interactions produce the turbulence. A related possibility is that the scintillations occur where the magnetic fields of the S1 and $\mathrm{S} 2$ shells interact.

\section{Conclusions}

The IBEX mission is now providing detailed in situ measurements of ENAs formed in the interface between the CHISM and the solar wind. The ENA data will constrain the region where the filtration of interstellar neutrals occurs. The advent of observations of ISMa inside of the heliosphere brought an absolutely unique perspective to understanding interstellar clouds. Observations of ISMa inside and outside of the heliosphere can be combined with extraordinary accuracy to constrain the questions concerning the boundary of the heliosphere. We find that the CHISM properties consistent with available heliospheric and astronomical data on the LIC indicate a warm, low-density, partially ionized gas, $n\left(\mathrm{H}^{\circ}\right) \sim 0.195 \mathrm{~cm}^{-3}, n(\mathrm{e}) \sim 0.06 \mathrm{~cm}^{-3}, T \sim 6300 \mathrm{~K}, \chi(\mathrm{H}) \sim 0.24, \chi(\mathrm{He}) \sim 0.40$. If thermal and magnetic pressures are equal, the magnetic field is weak, with $B \sim 2.7 \mu \mathrm{G}$.

This chapter in the IBEX volume summarizes data on interstellar material inside and outside of the heliosphere, including $\mathrm{H}^{\circ}, \mathrm{He}^{\circ}$, and pickup ions and anomalous cosmic rays. Accurate corrections for filtration are the key to comparing data on interstellar neutrals inside of the heliosphere with data on interstellar neutrals in the CHISM. The energetic neutral atom data measured by IBEX will provide important data for constraining the heliosphere boundary regions and the filtration corrections for interstellar neutrals.

Consistent values for the orientation of the interstellar magnetic field at the heliosphere are given by several different indicators, and suggest a field inclined by $\sim 59^{\circ}$ with respect to the ecliptic plane; if the ISMF is part of the extended Loop I magnetic field, the field polarity may be directed from the southern to the northern hemisphere. Additional data on the polarizations of nearby stars would strengthen our understanding of the nearby interstellar magnetic field.

This chapter also provides the context for understanding the origin of the CHISM, and the family of cloudlets that surround the CHISM on our journey through space. Clouds such as the CHISM, e.g. warm, low-density, partially ionized gas, were once termed "intercloud medium". However the dynamical motion of the CHISM and other nearby clouds clearly indicate a common origin and history for a parcel of gas that moves through the LSR at a mean velocity of $\sim 17 \mathrm{~km} \mathrm{~s}^{-1}$. Recent studies of distinct kinematical components among the clump of nearby cloudlets offers the opportunity to understand cloud-cloud interactions, as 
well as the paleoheliosphere. Identifying and modeling CLIC kinematics requires grappling with the fundamental concept of 'what is an interstellar cloud', in the context of a turbulent flow, or superbubble shell, containing very low-density gas. The dust grains in the CHISM appear typical for rapidly moving clouds with a history of grain destruction in shocks, and at the same time in situ dust data provide a means of direct measurement of the interstellar gas-to-dust mass ratio. The small dust grains missing from the inner heliosphere are clues to the interstellar and solar wind magnetic fields in the heliosheath regions, and the way the interstellar dust distribution is modified by the radiation pressure and the solar activity cycle. The origin of the LIC as part of an expanding superbubble shell driven by star formation in the Scorpius-Centaurus Association is consistent with data on the LISM.

Summary of acronyms used in this chapter The many terms and acronyms used in this chapter include: Interstellar Boundary Explorer mission (IBEX), Local Bubble (LB), Voyager 1 (V1), Voyager 2 (V2), anomalous cosmic rays (ACR), circumheliospheric interstellar medium (CHISM), complex of local interstellar clouds (CLIC), energetic neutral atom (ENA), extreme ultraviolet (EUV), far ultraviolet (FUV), heliopause (HP), Hubble Space Telescope (HST), interplanetary magnetic field (IMF), interstellar dust grain (ISDG), interstellar magnetic field (ISMF), interstellar material (ISMa), interstellar radiation field (ISRF), Local Interstellar Cloud (LIC), local interstellar medium (LISM), local standard of rest (LSR), magnetohydrodynamic (MHD), pickup ion (PUI), polycyclic aromatic hydrocarbons (PAH), position angle (PA), solar wind charge exchange (SWCX), solar wind termination shock (TS), and ultraviolet (UV).

Acknowledgements P. Frisch, D. McComas, E. Möbius and N. Schwadron gratefully acknowledge support from NASA through the IBEX Explorer mission. P. Frisch thanks NASA for support through grants NNG06GE33G and NNX08AJ33G. We would like to acknowledge Dimitra Koutroumpa for kindly sharing her SWCX spectrum with us in advance of the publication of her article. S. Redfield would like to acknowledge support provided by NASA through Hubble Fellowship grant HST-HF-01190.01 awarded by the Space Telescope Science Institute, which is operated by the Association of Universities for Research in Astronomy, Inc., for NASA, under contract NAS 5-26555.

\section{References}

T.F. Adams, P.C. Frisch, Astrophys. J. 212, 300-308 (1977)

J.M. Ajello, A.I. Stewart, G.E. Thomas, A. Graps, Astrophys. J. 317, 964-986 (1987)

D.B. Alexashov, S.V. Chalov, A.V. Myasnikov, V.V. Izmodenov, R. Kallenbach, Astron. Astrophys. 420, 729-736 (2004)

N. Altobelli, S. Kempf, M. Landgraf, R. Srama, V. Dikarev, H. Krüger, G. Moragas-Klostermeyer, E. Grün, J. Geophys. Res. (Space Phys.) 108, 7-1 (2003)

N. Altobelli, S. Kempf, H. Krüger, M. Landgraf, M. Roy, E. Grün, J. Geophys. Res. (Space Phys.) 110, 7102 (2005)

N. Altobelli, E. Grün, M. Landgraf, Astron. Astrophys. 448, 243-252 (2006)

K. Avinash, J. Slavin, G.P. Zank, P. Frisch, AGU Fall Meeting Abstracts pages B1602+ (2008)

W.I. Axford, in The Solar Wind, ed. by J.M.W.C.P. Sonnet, P.J. Coleman. NASA Spec. Publ., vol. 308 (NASA, Washington, 1972), p. 609

W.J. Baggaley, J. Geophys. Res. 105, 10,353-10,362 (2000)

M. Baguhl, E. Grün, D.P. Hamilton, G. Linkert, R. Riemann, P. Staubach, H.A. Zook, Space Sci. Rev. 72, 471-476 (1995)

V.B. Baranov, Y.G. Malama, J. Geophys. Res. 98, 15,157-15,163 (1993)

V.B. Baranov, Y.G. Malama, J. Geophys. Res. 100, 14,755-14,761 (1995)

V.B. Baranov, M.G. Lebedev, Y.G. Malama, Astrophys. J. 375, 347-351 (1991)

V.B. Baranov, V.V. Izmodenov, Y.G. Malama, J. Geophys. Res. 103, 9575-9585 (1998)

E.M. Berkhuijsen, Astron. Astrophys. 14, 359-386 (1971) 
J.L. Bertaux, J.E. Blamont, Astron. Astrophys. 11, 200 (1971)

N.G. Bochkarev, Astrophys. Space Sci. 138, 229-302 (1987)

D. Breitschwerdt, M.A. de Avillez, Astron. Astrophys. 452, L1-L5 (2006)

D. Breitschwerdt, M.J. Freyberg, R. Egger, Astron. Astrophys. 361, 303-320 (2000)

L.F. Burlaga, N.F. Ness, M.H. Acuña, R.P. Lepping, J.E.P. Connerney, J.D. Richardson, Nature 454, 75-77 (2008)

M. Bzowski, Acta Astron. 38, 443-453 (1988)

M. Bzowski, in The Outer Heliosphere: The Next Frontiers, ed. by K. Scherer, H. Fichtner, H.J. Fahr, E. Marsch. COSPAR Colloquia Series, vol. 11 (Elsevier/Pergamon, Amsterdam, 2001), pp. 69-72

M. Bzowski, H.J. Fahr, D. Ruciński, H. Scherer, Astron. Astrophys. 326, 396-411 (1997)

M. Bzowski, E. Möbius, S. Tarnopolski, V. Izmodenov, G. Gloeckler, Astron. Astrophys. 491, 7-19 (2008)

E. Chassefiere, J.L. Bertaux, R. Lallemant, V.G. Kurt, Astron. Astrophys. 160, 229-242 (1986)

J.T. Clarke, R. Lallement, J. Bertaux, H. Fahr, E. Quemerais, H. Scherer, Astrophys. J. 499, 482-488 (1998)

M.R. Collier, T.E. Moore, D. Simpson, A. Roberts, A. Szabo, S.A. Fuselier, P. Wurz, M.A. Lee, B.T. Tsurutani, Adv. Space Res. 34, 166-171 (2004)

D.P. Cox, L. Helenius, Astrophys. J. 583, 205-228 (2003)

T.E. Cravens, Astrophys. J. Lett. 532, L153-L156 (2000)

I.A. Crawford, Mon. Not. R. Astron. Soc. 327, 841-848 (2001)

I.A. Crawford, S.K. Dunkin, Mon. Not. R. Astron. Soc. 273, 219-224 (1995)

R.M. Crutcher, Astrophys. J. 254, 82-87 (1982)

A.C. Cummings, E.C. Stone, Space Sci. Rev. 130, 389-399 (2007)

A.C. Cummings, E.C. Stone, C.D. Steenberg, Astrophys. J. 578, 194-210 (2002)

A. Czechowski, I. Mann, Astron. Astrophys. 410, 165-173 (2003a)

A. Czechowski, I. Mann, J. Geophys. Res. 108, 13 (2003b)

E.J. de Geus, P.T. de Zeeuw, J. Lub, Astron. Astrophys. 216, 44-61 (1989)

R.J. Egger, B. Aschenbach, Astron. Astrophys. 294, L25-L28 (1995)

H.J. Fahr, Space Sci. Rev. 15, 483-540 (1974)

H.J. Fahr, Astron. Astrophys. 241, 251-259 (1991)

H.J. Fahr, T. Kausch, H. Scherer, Astron. Astrophys. 357, 268-282 (2000)

H.J. Fahr, H. Fichtner, K. Scherer, O. Stawicki, in Solar Journey: The Significance of Our Galactic Environment for the Heliosphere and Earth, ed. by P. Frisch (Springer, Berlin, 2006), pp. 259-279

L.A. Fisk, B. Kozlovsky, R. Ramaty, Astrophys. J. 190, L35-L38 (1974)

V. Florinski, G.P. Zank, in Solar Journey: The Significance of Our Galactic Environment for the Heliosphere and Earth, ed. by P. Frisch (Springer, Berlin, 2006), pp. 281-316

P.C. Frisch, Nature 293, 377-379 (1981)

P.C. Frisch, Science 265, 1423 (1994)

P.C. Frisch, Space Sci. Rev. 72, 499-592 (1995)

P.C. Frisch, Space Sci. Rev. 78, 213-222 (1996)

P.C. Frisch, Am. Sci. 88, 52-59 (2000)

P.C. Frisch, Introduction: The Paleoheliosphere versus PaleoLISM, in Solar Journey: The Significance of Our Galactic Environment for the Heliosphere and Earth, ed. by P.C. Frisch (Springer, Berlin, 2006), pp. $1-22$

P.C. Frisch, ArXiv e-prints: arXiv:0707.2970 (2007)

P.C. Frisch, Space Sci. Rev. 100 (2008a)

P.C. Frisch, Astrophys. J. (2008b, submitted). arXiv:0804.1901

P. Frisch, D.G. York, in The Galaxy and the Solar System (University of Arizona Press, Tucson, 1986), pp. 83-100

P.C. Frisch, J.D. Slavin, Short term variations in the galactic environment of the Sun, in Solar Journey: The Significance of Our Galactic Environment for the Heliosphere and Earth, ed. by P.C. Frisch (Springer, Berlin, 2006a), pp. 133-193

P.C. Frisch, J.D. Slavin, Astrophys. Space Sci. Trans. 2, 53-61 (2006b)

P.C. Frisch, D.E. Welty, D.G. York, J.R. Fowler, Astrophys. J. 357, 514-523 (1990)

P.C. Frisch, J.M. Dorschner, J. Geiss, J.M. Greenberg, E. Grün, M. Landgraf, P. Hoppe, A.P. Jones, W. Krätschmer, T.J. Linde, G.E. Morfill, W. Reach, J.D. Slavin, J. Svestka, A.N. Witt, G.P. Zank, Astrophys. J. 525, 492-516 (1999)

P.C. Frisch, L. Grodnicki, D.E. Welty, Astrophys. J. 574, 834-846 (2002)

B. Fuchs, D. Breitschwerdt, M.A. de Avillez, C. Dettbarn, C. Flynn, Mon. Not. R. Astron. Soc. 373, 993-1003 (2006)

K.G. Gayley, G.P. Zank, H.L. Pauls, P.C. Frisch, D.E. Welty, Astrophys. J. 487, 259-270 (1997)

J. Geiss, G. Gloeckler, L.A. Fisk, R. von Steiger, J. Geophys. Res. 100, 23373-23378 (1995)

G. Gloeckler, J. Geiss, Adv. Space Res. 34, 53-60 (2004) 
G. Gloeckler, L. Fisk, Composition of Matter. Space Sciences Series of ISSI, vol. 27 (Springer, Berlin, 2007), pp. $489-513$

G. Gloeckler, K.-P. Wenzel, Acceleration Processes of Heliospheric Particle Populations. Century of Space Science, vol. II (Kluwer Academic, Dordrecht, 2001), pp. 963-1006

G. Gloeckler, L.A. Fisk, J. Geiss, N.A. Schwadron, T.H. Zurbuchen, J. Geophys. Res. 105, 7459-7464 (2000)

G. Gloeckler, E. Möbius, J. Geiss, M. Bzowski, S. Chalov, H. Fahr, D.R. McMullin, H. Noda, M. Oka, D. Ruciński, R. Skoug, T. Terasawa, R. von Steiger, A. Yamazaki, T. Zurbuchen, Astron. Astrophys. 426, 845-854 (2004)

P.M. Gondhalekar, A.P. Phillips, R. Wilson, Astron. Astrophys. 85, 272 (1980)

K. Grogan, S.F. Dermott, B.A.S. Gustafson, S. Jayaraman, Y.L. Xu, D. Hamilton, in IAU Colloq. 150: Physics, Chemistry, and Dynamics of Interplanetary Dust, ed. by B.A.S. Gustafson, M.S. Hanner. Astronomical Society of the Pacific Conference Series, vol. 104 (Astronomical Society of the Pacific, San Francisco, 1996), p. 325

E. Grün, B. Gustafson, I. Mann, M. Baguhl, G.E. Morfill, P. Staubach, A. Taylor, H.A. Zook, Astron. Astrophys. 286, 915-924 (1994)

E. Grün, P. Staubach, M. Baguhl, D.P. Hamilton, H.A. Zook, S. Dermott, B.A. Gustafson, H. Fechtig, J. Kissel, D. Linkert, G. Linkert, R. Srama, M.S. Hanner, C. Polanskey, M. Horanyi, B.A. Lindblad, I. Mann, J.A.M. McDonnell, G.E. Morfill, G. Schwehm, Icarus 129, 270-288 (1997)

E. Grün, R. Srama, H. Krüger, S. Kempf, V. Dikarev, S. Helfert, G. Moragas-Klostermeyer, Icarus 174, 1-14 (2005)

C. Gry, E.B. Jenkins, Astron. Astrophys. 367, 617-628 (2001)

D.A. Gurnett, W.S. Kurth, Nature 454, 78-80 (2008)

D.A. Gurnett, W.S. Kurth, I.H. Cairns, J. Mitchell, in Physics of the Inner Heliosheath, ed. by J. Heerikhuisen, V. Florinski, G.P. Zank, N.V. Pogorelov. American Institute of Physics Conference Series, vol. 858 (Springer, New York, 2006), pp. 129-134

J.L. Han, Chin. J. Astron. Astrophys. Suppl. 6, 211-217 (2006)

J. Heerikhuisen, V. Florinski, G.P. Zank, J. Geophys. Res. 111(A10), 6110 (2006)

J. Heerikhuisen, N.V. Pogorelov, G.P. Zank, V. Florinski, Astrophys. J. Lett. 655, L53-L56 (2007)

J. Heerikhuisen, N.V. Pogorelov, V. Florinski, G.P. Zank, J.A. le Roux, Astrophys. J. 682 , 679-689 (2008)

D.B. Henley, R.L. Shelton, K.D. Kuntz, Astrophys. J. 661, 304-319 (2007)

L.M. Hobbs, Astrophys. J. 157, 135 (1969)

T.E. Holzer, J. Geophys. Res. 77, 5407-5431 (1972)

T.E. Holzer, Annu. Rev. Astron. Astrophys. 27, 199-234 (1989)

V. Izmodenov, B.E. Wood, R. Lallement, J. Geophys. Res. (Space Phys.) 107, 1308 (2002)

V. Izmodenov, Y. Malama, G. Gloeckler, J. Geiss, Astron. Astrophys. 414, L29-L32 (2004)

V. Izmodenov, D. Alexashov, A. Myasnikov, Astron. Astrophys. 437, L35-L38 (2005)

V.V. Izmodenov, Astrophys. Space Sci. 274, 55-69 (2000)

V.V. Izmodenov, Space Sci. Rev. 97, 385-388 (2001)

V.V. Izmodenov, D.B. Alexashov, Astron. Lett. 29, 58-63 (2003)

V.V. Izmodenov, J. Geiss, R. Lallement, G. Gloeckler, V.B. Baranov, Y.G. Malama, J. Geophys. Res. 104, 4731-4741 (1999a)

V.V. Izmodenov, J. Geiss, R. Lallement, G. Gloeckler, V.B. Baranov, Y.G. Malama, J. Geophys. Res. 104, 4731-4742 (1999b)

V.V. Izmodenov, M.A. Gruntman, Y.G. Malama, J. Geophys. Res. 106, 10,681-10,689 (2001)

A.P. Jones, A.G.G.M. Tielens, D.J. Hollenbach, C.F. McKee, Astrophys. J. 433, 797-810 (1994)

W.-T. Kim, E.C. Ostriker, Astrophys. J. 570, 132-151 (2002)

D. Koutroumpa, R. Lallement, V. Kharchenko, A. Dalgarno, ArXiv e-prints 805 (2008)

H. Krüger, N. Altobelli, B. Anweiler, S.F. Dermott, V. Dikarev, A.L. Graps, E. Grün, B.A. Gustafson, D.P. Hamilton, M.S. Hanner, M. Horányi, J. Kissel, M. Landgraf, B.A. Lindblad, D. Linkert, G. Linkert, I. Mann, J.A.M. McDonnell, G.E. Morfill, C. Polanskey, G. Schwehm, R. Srama, H.A. Zook, Planet. Space Sci. 54, 932-956 (2006)

H. Krüger, M. Landgraf, N. Altobelli, E. Grün, Space Sci. Rev. 130, 401-408 (2007)

W.S. Kurth, D.A. Gurnett, J. Geophys. Res. 108, 2-16 (2003)

R. Lallement, P. Bertin, Astron. Astrophys. 266, 479-485 (1992)

R. Lallement, R. Ferlet, Astron. Astrophys. 324, 1105-1114 (1997)

R. Lallement, A. Vidal-Madjar, R. Ferlet, Astron. Astrophys. 168, 225-236 (1986)

R. Lallement, R. Ferlet, A.M. Lagrange, M. Lemoine, A. Vidal-Madjar, Astron. Astrophys. 304, 461-474 (1995)

R. Lallement, E. Quémerais, J.L. Bertaux, S. Ferron, D. Koutroumpa, R. Pellinen, Science 307, 1447-1449 (2005)

M. Landgraf, J. Geophys. Res. 105, 10,303-10,316 (2000) 
M. Landgraf, E. Grün, in IAU Colloq. 166: The Local Bubble and Beyond, ed. by D. Breitschwerdt, M.J. Freyberg, J. Truemper. Lecture Notes in Physics, vol. 506 (Springer, Berlin, 1998), pp. 381-384

M. Landgraf, K. Augustsson, E. Grün, B.Å.S. Gustafson, Science 286, 2319-2322 (1999)

M. Landgraf, W.J. Baggaley, E. Grün, H. Krüger, G. Linkert, J. Geophys. Res. 105, 10343-10352 (2000)

M. Landgraf, H. Krüger, N. Altobelli, E. Grün, J. Geophys. Res. (Space Phys.) 108, 5-1 (2003)

N. Lehner, E. Jenkins, C. Gry, H. Moos, P. Chayer, S. Lacour, Astrophys. J. 595, 858-879 (2003)

T.J. Linde, T.I. Gombosi, J. Geophys. Res. 105, 10,411-10,418 (2000)

T.J. Linde, T.I. Gombosi, P.L. Roe, K.G. Powell, D.L. DeZeeuw, J. Geophys. Res. 103, 1889-1904 (1998)

J.L. Linsky, B.E. Wood, Astrophys. J. 463, 254-270 (1996)

J.L. Linsky, B.J. Rickett, S. Redfield, Astrophys. J. 675, 413-419 (2008)

K. Lodders, Astrophys. J. 591, 1220-1247 (2003)

P.B. Lucke, Astron. Astrophys. 64, 367-377 (1978)

E. Möbius, M. Bzowski, S. Chalov, H.-J. Fahr, G. Gloeckler, V. Izmodenov, R. Kallenbach, R. Lallement, D. McMullin, H. Noda, M. Oka, A. Pauluhn, J. Raymond, D. Ruciński, R. Skoug, T. Terasawa, W. Thompson, J. Vallerga, R. von Steiger, M. Witte, Astron. Astrophys. 426, 897-907 (2004)

H.-R. Müller, G.P. Zank, J. Geophys. Res. 7104-7116 (2004)

J. Maíz-Apellániz, Astrophys. J. Lett. 560, L83-L86 (2001)

Y.G. Malama, V.V. Izmodenov, S.V. Chalov, Astron. Astrophys. 445, 693-701 (2006)

I. Mann, H. Kimura, J. Geophys. Res. 105, 10,317-10,328 (2000)

L.A. Marschall, L.M. Hobbs, Astrophys. J. 173, 43-62 (1972)

J.S. Mathis, W. Rumpl, K.H. Nordsieck, Astrophys. J. 217, 425-433 (1977)

W. McClintock, R.C. Henry, H.W. Moos, J.L. Linsky, Astrophys. J. Lett. 204, L103-L106 (1976)

D.J. McComas, N.A. Schwadron, Geophys. Res. Lett. 33, 4102 (2006)

D. McComas, F. Allegrini, P. Bochsler, M. Bzowski, M. Collier, H. Fahr, H. Fichtner, P. Frisch, H. Funsten, S. Fuselier, G. Gloeckler, M. Gruntman, V. Izmodenov, P. Knappenberger, M. Lee, S. Livi, D. Mitchell, E. Möbius, T. Moore, D. Reisenfeld, E. Roelof, N. Schwadron, M. Wieser, M. Witte, P. Wurz, G. Zank, in AIP Conf. Proc. 719: Physics of the Outer Heliosphere, pp. 162-181 (2004a)

D.J. McComas, N.A. Schwadron, F.J. Crary, H.A. Elliott, D.T. Young, J.T. Gosling, M.F. Thomsen, E. Sittler, J.-J. Berthelier, K. Szego, A.J. Coates, J. Geophys. Res. (Space Phys.) 109, 2104 (2004b)

D. McComas, F. Allegrini, L. Bartolone, P. Bochsler, M. Bzowski, M. Collier, H. Fahr, H. Fichtner, P. Frisch, H. Funsten, S. Fuselier, G. Gloeckler, M. Gruntman, V. Izmodenov, P. Knappenberger, M. Lee, S. Livi, D. Mitchell, E. Möbius, T. Moore, S. Pope, D. Reisenfeld, E. Roelof, H. Runge, J. Scherrer, N. Schwadron, R. Tyler, M. Wieser, M. Witte, P. Wurz, G. Zank, in Solar Wind 11/SOHO 16, Connecting Sun and Heliosphere. ESA Special Publication, vol. 592, pp. 689-692 (2005)

D.J. McComas, F. Allegrini, L. Bartolone, P. Bochsler, M. Bzowski, M. Collier, H. Fahr, H. Fichtner, P. Frisch, H. Funsten, S. Fuselier, G. Gloeckler, M. Gruntman, V. Izmodenov, P. Knappenberger, M. Lee, S. Livi, D. Mitchell, E. Möbius, T. Moore, S. Pope, D. Reisenfeld, E. Roelof, H. Runge, J. Scherrer, N. Schwadron, R. Tyler, M. Wieser, M. Witte, P. Wurz, G. Zank, in Physics of the Inner Heliosheath, ed. by J. Heerikhuisen, V. Florinski, G.P. Zank, N.V. Pogorelov. American Institute of Physics Conference Series, vol. 858 (Springer, Berlin, 2006), pp. 241-250

D.J. McComas et al., Space Sci. Rev. (2009, this issue)

J.J. Mitchell, I.H. Cairns, H.-R. Müller, G.P. Zank, Geophys. Res. Lett. 32, 7101 (2005)

E. Möbius, D. Hovestadt, B. Klecker, M. Scholer, G. Gloeckler, Nature 318, 426-429 (1985)

H.-R. Müller, G.P. Zank, A.S. Lipatov, J. Geophys. Res. 105, 27,419-27,438 (2000)

H.-R. Müller, P.C. Frisch, V. Florinski, G.P. Zank, Astrophys. J. 647, 1491-1505 (2006)

H.-R. Müller, V. Florinski, J. Heerikhuisen, V.V. Izmodenov, K. Scherer, D. Alexashov, H. Fahr, Astron. Astrophys. 491, 43-51 (2008)

G. Munch, A. Unsold, Astrophys. J. 135, 711-715 (1962)

W.R. Oegerle, E.B. Jenkins, R.L. Shelton, D.V. Bowen, P. Chayer, Astrophys. J. 622, 377-389 (2005)

M. Opher, E.C. Stone, T.I. Gombosi, Science 316, 875 (2007)

M. Opher, E.C. Stone, G. Toth, V. Izmodenov, V. Alexashov, T.I. Gombosi, AGU Fall Meeting Abstracts page A7 (2008)

V. Piirola, Astron. Astrophys. Suppl. Ser. 30, 213 (1977)

N. Pogorelov, T. Matsuda, J. Geophys. Res. 103, 237 (1998)

N.V. Pogorelov, G.P. Zank, T. Ogino, Astrophys. J. 614, 1007-1021 (2004)

N.V. Pogorelov, G.P. Zank, T. Ogino, Astrophys. J. 644, 1299-1316 (2006)

N.V. Pogorelov, E.C. Stone, V. Florinski, G.P. Zank, Astrophys. J. 668, 611-624 (2007)

N.V. Pogorelov, J. Heerikhuisen, G.P. Zank, Astrophys. J. Lett. 675, L41-L44 (2008a)

N.V. Pogorelov, G.P. Zank, T. Ogino, Adv. Space Res. 41, 306-317 (2008b)

E. Quémerais, V. Izmodenov, Astron. Astrophys. 396, 269-281 (2002) 
E. Quémerais, R. Lallement, S. Ferron, D. Koutroumpa, J.-L. Bertaux, E. Kyrölä, W. Schmidt, J. Geophys. Res. 111, A10/9114 (2006)

R. Ratkiewicz, A. Barnes, G.A. Molvik, J.R. Spreiter, S.S. Stahara, M. Vinokur, S. Venkateswaran, Astron. Astrophys. 335, 363-369 (1998)

S. Redfield, R.E. Falcon, Astrophys. J. 683, 207-225 (2008)

S. Redfield, J.L. Linsky, Astrophys. J. 551, 413-428 (2001)

S. Redfield, J.L. Linsky, Astrophys. J. Suppl. Ser. 139, 439-465 (2002)

S. Redfield, J.L. Linsky, Astrophys. J. 602, 776-802 (2004a)

S. Redfield, J.L. Linsky, Astrophys. J. 613, 1004-1022 (2004b)

S. Redfield, J.L. Linsky, Astrophys. J. 673, 283-314 (2008)

J.D. Richardson, Y. Liu, C. Wang, Adv. Space Res. 41, 237-244 (2008)

I.P. Robertson, T.E. Cravens, J. Geophys. Res. 108, 6-1 (2003)

J.B. Rogerson, D.G. York, J.F. Drake, E.B. Jenkins, D.C. Morton, L. Spitzer, Astrophys. J. Lett. 181, L110L114 (1973)

P. Routly, J.L. Spitzer, Astrophys. J. 115, 227 (1952)

D. Ruciński, M. Bzowski, Astron. Astrophys. 296, 248-263 (1995)

D. Ruciński, H.J. Fahr, S. Grzȩdzielski, Planet. Space Sci. 41, 773-783 (1993)

D. Ruciński, A.C. Cummings, G. Gloeckler, A.J. Lazarus, E. Mobius, M. Witte, Space Sci. Rev. 78, 73-84 (1996)

D. Russeil, Astron. Astrophys. 397, 133-146 (2003)

E. Salerno, F. Bühler, P. Bochsler, H. Busemann, M.L. Bassi, G.N. Zastenker, Y.N. Agafonov, N.A. Eismont, Astrophys. J. 585, 840-849 (2003)

B.D. Savage, N. Lehner, Astrophys. J. Suppl. Ser. 162, 134-160 (2006)

N.A. Schwadron, G. Gloeckler, Space Sci. Rev. 130, 283-291 (2007)

N.A. Schwadron, D.J. McComas, Geophys. Res. Lett. 34, 14,105 (2007)

N.A. Schwadron, M.A. Lee, D.J. McComas, Astrophys. J. 675, 1584-1600 (2008)

D.M. Sfeir, R. Lallement, F. Crifo, B.Y. Welsh, Astron. Astrophys. 346, 785-797 (1999)

J.D. Slavin, Astrophys. J. 346, 718-727 (1989)

J.D. Slavin, P.C. Frisch, Astrophys. J. Lett. 651, 37 (2006)

J.D. Slavin, P.C. Frisch, Astron. Astrophys. 491, 53-68 (2008)

S.L. Snowden, M.J. Freyberg, K.D. Kuntz, W.T. Sanders, Astrophys. J. Suppl. Ser. 128, 171-212 (2000)

L. Spitzer, Physical Processes in the Interstellar Medium (Wiley, New York, 1978)

E.C. Stone, A.C. Cummings, F.B. McDonald, B.C. Heikkila, N. Lal, W.R. Webber, Science 309, 2017-2020 (2005)

E.C. Stone, A.C. Cummings, F.B. McDonald, B.C. Heikkila, N. Lal, W.R. Webber, Nature 454, 71-74 (2008)

G.E. Thomas, Ann. Rev. Earth Planet. Sci. 6, 173-204 (1978)

G.E. Thomas, R.F. Krassa, Astron. Astrophys. 11, 218-233 (1971)

J. Tinbergen, Astron. Astrophys. 105, 53-64 (1982)

J. Vallerga, Space Sci. Rev. 78, 277-288 (1996)

J. Vallerga, Astrophys. J. 497, 921-927 (1998)

J. Vallerga, R. Lallement, M. Lemoine, F. Dalaudier, D. McMullin, Astron. Astrophys. 426, 855-865 (2004) J.V. Vallerga, P.W. Vedder, N. Craig, B.Y. Welsh, Astrophys. J. 411, 729-749 (1993)

M.K. Wallis, Nature 254, 202 (1975)

J.C. Weingartner, B.T. Draine, Astrophys. J. Suppl. Ser. 134, 263-281 (2001)

C.S. Weller, R.R. Meier, Astrophys. J. 193, 471-476 (1974)

C.S. Weller, R.R. Meier, Astrophys. J. 246, 386-393 (1981)

B.Y. Welsh, Space Sci. Rev. 57 (2008)

D.E. Welty, L.M. Hobbs, Astrophys. J. Suppl. Ser. 133, 345-393 (2001)

D.E. Welty, L.M. Hobbs, J.T. Lauroesch, D.C. Morton, L. Spitzer, D.G. York, Astrophys. J. Suppl. Ser. 124, 465-501 (1999)

D.E. Welty, L.M. Hobbs, D.C. Morton, Astrophys. J. Suppl. Ser. 147, 61-96 (2003)

D.E. Welty, D.C. Morton, L.M. Hobbs, Astrophys. J. Suppl. Ser. 106, 533 (1996a)

D.E. Welty, D.C. Morton, L.M. Hobbs, Astrophys. J. Suppl. Ser. 106, 533-562 (1996b)

M.E. Wiedenbeck, W.R. Binns, A.C. Cummings, A.J. Davis, G.A. de Nolfo, M.H. Israel, R.A. Leske, R.A. Mewaldt, E.C. Stone, T.T. von Rosenvinge, Space Sci. Rev. 130, 415-429 (2007)

M. Witte, Astron. Astrophys. 426, 835-844 (2004)

B. Wolff, D. Koester, R. Lallement, Astron. Astrophys. 346, 969-978 (1999)

M. Wolleben, Astrophys. J. 664, 349-356 (2007)

B.E. Wood, C.W. Ambruster, A. Brown, J.L. Linsky, Astrophys. J. 542, 411-420 (2000a)

B.E. Wood, J.L. Linsky, G.P. Zank, Astrophys. J. 537, 304-311 (2000b)

B.E. Wood, S. Redfield, J.L. Linsky, H.-R. Müller, G.P. Zank, Astrophys. J. Suppl. Ser. 159, 118-140 (2005) 
B.E. Wood, V.V. Izmodenov, J.L. Linsky, Y.G. Malama, Astrophys. J. 657, 609-617 (2007)

B.E. Wood, V.V. Izmodenov, Y.G. Malama, Space Sci. Rev. 75 (2008)

P. Wurz, M.R. Collier, T.E. Moore, D. Simpson, S. Fuselier, W. Lennartson, in Physics of the Outer Heliosphere, ed. by V. Florinski, N.V. Pogorelov, G.P. Zank. American Institute of Physics Conference Series, vol. 719 (Springer, Berlin, 2004), pp. 195-200

D.G. York, B.F. Kinahan, Astrophys. J. 228, 127-146 (1979)

G.P. Zank, Space Sci. Rev. 89, 413-688 (1999)

G.P. Zank, H.L. Pauls, L.L. Williams, D. Hall, J. Geophys. Res. 101, 21,636-21,655 (1996) 